\title{
Efficient Auction Mechanisms for Supply Chain Procurement
}

\author{
Rachel R. Chen \\ Anderson Graduate School of Management, University of California, \\ Riverside, California 92521, rachel.chen@ucr.edu \\ Robin O. Roundy \\ School of ORIE, Cornell University, Ithaca, New York 14853, robin@orie.cornell.edu \\ Rachel Q. Zhang \\ Department of Industrial Engineering and Engineering Management \\ Hong Kong University of Science and Technology, \\ Clear Water Bay, Kowloon, Hong Kong, rzhang@ust.hk \\ Ganesh Janakiraman \\ Stern School of Business, New York University, New York, New York 10012, \\ gjanakir@stern.nyu.edu
}

\begin{abstract}
We consider multi-unit Vickrey auctions for procurement in supply chain settings. This is the first paper that incorporates transportation costs into auctions in a complex supply network. We first introduce an auction mechanism that makes simultaneous production and transportation decisions so that the total supply chain cost is minimized and induces truthtelling from the suppliers. Numerical study shows that considerable supply chain cost savings can be achieved if production and transportation costs are considered simultaneously. However, the buyer's payments in such auctions can be high. We then develop a new Vickrey type auction that incorporates the buyer's reservation price function into quantity allocation and payment decision. As a result, the buyer has some control over his payments at the expense of introducing uncertainty in the quantity acquired in the auction.
\end{abstract}

\section{Introduction}

Research and practice in operations management has emphasized integrated supply chains, where long-term relationships between suppliers and buyers of manufactured components facilitate optimization of total supply chain costs. There is a recent emphasis on using auctions for supply chain procurement (Chin 2003), especially for non-critical components. It has become an effective and efficient means of achieving lower acquisition costs, lower barriers for new suppliers to enter a market, and consequently better market efficiency. With recent advances in information technology, such auctions can be carried out through the Internet, referred to as online auctions. Online auctions allow geographically diverse buyers and sellers to exchange goods, services, and information, and to dynamically determine prices that reflect the demand and supply at a certain point of time so 
that efficient matching of supply and demand can be realized. As pointed out by Lucking-Reiley (2000), online auctions often lead to lower information, transaction and participation costs, as well as increased convenience for both sellers and buyers, the ability for asynchronous bidding, and easier access to larger markets.

Most auctions are price-driven. However, there are other costs associated with integrating a supply chain, e.g., costs associated with transportation, capacity management, inventories, etc. Recently, there have been escalating disputes in the electronics industry on fair pricing in supply chains. According to industry executives, OEMs (Original Equipment Manufacturers) and suppliers are ignoring the numerous additional costs that affect parts prices, such as shipping, logistics, and value-added services when they quote prices. They are passing such costs onto their manufacturing partners and distributors, squeezing profitability out of the channel (Sullivan and Lamb 2001). According to Mike Dennison, senior director of Global Procurement and Strategic Supply Chain Management at Flextronics International, "Suppliers must understand all the complexities of getting material from their dock to the OEM's and need to factor in the cost when they are quoting a price". This issue relates to more than a general understanding of terms and conditions, according to Andy Fischer, vice president and channel director for Avnet Electronics Marketing at Avnet Inc., "Continuity (of a supply chain) ... is not only channel related, it's also global. Service packages, demand curves, global locations all lead to different costs and requirements." As Chopra and Van Mieghem (2000) pointed out, accounting for these additional costs may be a key issue in Business-to-Business auctions. Yet, there is a lack of decision analysis that combines sophisticated Operations Research/Management Science (OR/MS) tools with economic insights to help supply chain practitioners in auctions and e-marketplaces (Keskinocak and Tayur 2001).

The goals of this paper are to design and analyze multi-unit auctions that achieve allocative efficiency by taking into account both production and transportation costs that have been mostly ignored in the auction literature, and to illustrate some of the risks and tradeoffs associated with these auctions. We consider a single round sealed bid procurement (reverse) auction organized by an independent auctioneer. There is a single buyer who has demand for a single unit or multiple units of a product at a set of geographically diverse locations. There are several potential suppliers (or sellers) in the market; each owns multiple production locations and has her own cost structure. (Note that all the analyses in this paper can be applied to a forward auction with a single seller and multiple buyers, for which similar results will hold). To participate in the auction, each supplier is required to submit a bid (e.g., a price schedule) to the auctioneer, without observing other suppliers' 
bids (sealed bid). The auctioneer receives bids from suppliers and determines the quantities to purchase from and the payments to each supplier through some pre-specified rule known as an auction mechanism. The buyer makes his payments to the suppliers plus the transportation costs. A mechanism is truthful (or induces truth-telling) if for every supplier truth-telling is a dominant strategy (Nisan and Ronen 2001). Truth-telling from all the suppliers is important because it is usually a requirement for a multi-unit auction to be allocatively efficient. Traditionally, allocative efficiency refers to a condition achieved when the buyer's business is awarded to a set of suppliers with the lowest total production cost. In our paper, we extend the definition to include both the production costs and transportation costs. That is, we refer allocatively efficient auctions to be those that award contracts to suppliers with the minimum total production and transportation cost (i.e., achieve the lowest total supply chain cost). For simplicity, we refer allocatively efficient auctions as efficient auctions throughout the paper. Note that the total amount exchanged in an efficient auction may not necessarily maximize the supply chain profit.

We focus on auctions organized by a credible third party auctioneer (e.g., FreeMarkets, eBreviate), whose existence provides incentives for suppliers to participate and bid competitively in an auction. Since the independent auctioneer is more interested in the long run health and growth of his business, rather than his commission revenue from a single auction (Rothkopf and Harstad 1994), we do not design auctions from the buyer's perspective. Instead, we propose efficient auction mechanisms as market instruments that can better match demand with supply, which is consistent with the trend of supply chain integration today. For example, Covisint.com, the leader in the automotive industry for online exchange, provides auction services (recently aquired by FreeMarkets) and "the goal (of the exchange) was integration and collaboration, promising lower cost, easier business practices and a market increase in efficiencies for the entire industry" (www.covisint.com). There are many ways that the auctioneer can bring in revenues. For procurement auctions, one option is to charge each buyer an annual subscription fee (e.g., covisint.com). In return, buyers can hold auctions as often as they want (Kisiel 2002). Other types of revenue for auctioneers include a commission on every transaction, or some fees charged for the auction service provided (software, training, technical support and consulting service, etc.). The anuual payment scheme is assumed in this paper, while the service is free to every supplier so as to attact a large supplier base.

We first propose an efficient auction mechanism (Auction $T$ ) which takes transportation costs into account while awarding supply chain contracts, and its payment structure induces suppliers to bid truthfully. To illustrate the importance of incorporating transportation costs into auctions in 
supply chain procurement, we examine an auction mechanism (Auction $S$ ), which awards contracts solely based on suppliers' production costs and is obviously not allocatively efficient. However, we show that the buyer may prefer Auction $S$ to Auction $T$ under certain conditions. This happens in manufacturing systems where per-unit costs tend to be relatively flat when the regular-time capacity of the manufacturing system is not exceeded, and to grow rapidly when overtime or other measures are required to increase capacity in the short term. In Auction $T$, such manufacturing cost structure can lead to high buyer payments to the suppliers. As a result, the buyer may prefer Auction $S$, simply because it reduces the size of these payments. We then introduce another efficient auction, Auction $R$, that allows the buyer a certain level of control over his payments, while still guarantees a healthy margin for the suppliers. That is, the suppliers always receive more than their offered bids so that the auction can attract a sufficient number of suppliers to participate. We show that the buyer will always pay less in Auction $R$ than in Auction $T$ at the expense of new risks associated with the quantities awarded, which are the outcome of the auction.

The contributions of our paper to the OR/MS literature can be summarized as follows.

1. Complex supply network We introduce optimization-based efficient auctions for the procurement of components in complex supply chain settings that involve multiple suppliers and buyer locations. The auctions are designed to account for both production and transportation costs which have been ignored in most auction literature. This opens the door to the solution of a host of supply chain optimization problems using auctions.

2. Multi-unit efficient auctions We consider multi-unit auctions that allow multiple winners, each of whom can be awarded multiple units at multiple production locations, while the majority of research on auctions so far limits its effort to either single-unit auctions or multiunit auctions in which each bidder can be awarded at most one unit. As a result, our auction mechanisms provide opportunities for available supply surplus across an entire industry to aggregate and meet certain demand in a complex supply chain network.

\section{Study on the benefits of incorporating transportation costs into supply chain} auctions We illustrate by numerical examples that considerable savings can be achieved if production and transportation costs are considered simultaneously while allocating contracts. However, we show that the buyer may prefer Auction $S$ which ignores the transportation costs while making allocation decisions to Auction T. This provides some important insights for auction design in supply chains. 


\section{Incorporate a (reported) reservation price function into payment determination}

High buyer payments in Auction $T$ is a major reason that Vickrey auctions are not widely used in practice, despite that they guarantee truthful bidding and efficient allocation. To solve this problem, we design Auction $R$, in which the buyer reports to the auctioneer a function representing his private valuation of every possible consumption level, from which his reservation price (the maximum amount the buyer is willing to pay) for every additional unit he acquires can be derived. While a flat reservation price is commonly used in auctions to give the buyer the option of walk away or to limit the buyer's payments, here a reservation price function is incorporated by the auctioneer to determine the payments to the suppliers. As a result, the buyer has some control over his total payment, at the expense of the uncertainty associated with the procurement quantities which are the outcome of the auction. Segal (2003) considered a multi-unit forward auction with one seller and multiple buyers, where each buyer can be awarded at most one unit. The seller's reservation supply curve can protect her from selling at a loss, and help maximize her revenue. However, so far there has been little discussion on whether and how the seller can achieve better revenue by submitting a reservation supply curve (which may not be the true one), especially in auctions where each winner can be awarded multiple units. Such possibilities are explored in Auction $R$. The reservation price function contains the demand signal, while bids from the suppliers reflect the supply condition. The buyer's payments, as functions of his reservation prices and the suppliers' bids, depend on the market condition when the procurement occurs. The risks and tradeoffs associated with the auction are illustrated through numerical examples.

In both Auctions $T$ and $R$, each supplier's dominant strategy is to bid at her true costs. Therefore, there is no need for suppliers to carry out market research and design bidding strategies. Under efficient allocations, such auctions are fair to all the suppliers. With the advances of artificial intelligence, such auctions can be carried out by virtual agents (e.g., user-customization, marketbased computation on a distributed computer network) (Nisan and Ronen 2001). Those agents receive bids, decide awards and payments based on some pre-specified algorithms designed not to reveal buyer's and suppliers' private information to the public. Furthermore, the Internet may make auctions accessible to bidders from all over the world, while computer agents can keep bidders from knowing each other, thus mitigate collusion. Rapid advances in computational capability make it now possible to conduct sophisticated allocation and payment rules (e.g., auction mechanisms in 
this paper) quickly and at low cost. Therefore, the auctions we propose in this paper have the potential to be successfully implemented online.

The paper is organized as follows. We discuss some of the relevant literature in Section 2, and introduce our models in Section 3. In Section 4, we introduce Auction $T$ and compare it with Auction $S$. In Section 5, we discuss payment reduction using Auction $R$ at the cost of uncertainty in the quantity purchased in the auction. The paper concludes in Section 6 .

\section{Literature Review}

Incorporating transportation costs into production and shipment decisions has been a rich research area in operations research. As an example, Sharp et al. (1970) consider a manufacturer who owns multiple production plants with non-linear production costs and provides products to multiple demand locations. The demands at different locations are known and the objective is to minimize the total production and transportation costs. When there is more than one production facility and production costs are non-linear, the common approach, which combines production and transportation costs on each arc of the supply network and makes production and transportation decisions based on a single cost function, does not apply. Recently, Holmberg and Tuy (1999) extend the classic transportation problem by allowing fixed costs of production at the supply nodes, and stochastic demand and convex non-linear penalty costs for being short at the demand centers. They reduce the problem to a simpler optimization problem and solve it using a branch and bound method. Both papers provide lists of references dealing with similar optimization problems. However, none of them considers pricing decisions using auctions.

Bidding behavior in auctions has been studied since the 1950's using game theory and decision theory (Rothkopf 1969). We refer the reader to Vickrey (1961), Milgrom and Weber (1982), Klemperer (1999), McAfee and McMillan (1987), and Rothkopf and Harstad (1994) for classical auction theory. According to Ausubel and Cramtom (1998), there are three dominant types of mechanisms for multi-unit auctions, known as Pay-as-You-Bid, uniform-price, and VCG auctions (attributed to Vickrey 1961, Clarke 1971, and Groves 1973, also known as Vickrey auctions or second-price auctions in the single-unit setting) The Pay-as-You-Bid auction is self-explanatory. In a uniform auction a uniform price is paid for each unit purchased. The price can be either the first rejected or the last accepted bid. In economics literature, it is well known that neither Pay-as-You-Bid nor uniform-price auctions is truth-inducing or efficient in general. (The only ex- 
ception is a uniform-price auction where each seller is limited to be awarded at most one unit.). The family of mechanisms known as VCG auctions is both truth-inducing and efficient. In a VCG auction, the buyer's payment to a seller is based not only on her bids, but also on the contribution that the seller makes to the system by participating in the auction. Therefore, truthful bidding is a dominant strategy for every bidder. The reader is referred to Nisan and Ronen (2001) for a summary and general definition of the VCG mechanism. In spite of the attractive properties of such auctions, they are not widely used in practice due to the fact that VCG payments may result in high buyer payments (Vickrey 1961). Also, human auctioneers may take advantage of the truth telling bidders and do not always act truthfully (Rothkopf et al. 1990). Furthermore, collusion among bidders can have a serious effect on the outcome of VCG type of auctions due to the way the payments are determined (we will describe in detail later). However, as we discussed in Introduction, a well-designed auction carried out online by independent virtual agents can prevent problems caused by human auctioneers and reduce the possibility of coalition formation. With our proposed Auction $R$, the buyer will have some control over his payments. All of these will make VCG family a practical form of auction mechanisms.

Recently, we have seen a growing body of literature on auctions in operations management. Cooper and Menich (1998) propose a method that belongs to the generalized VCG family for auction of airline tickets where bidders vie for different itineraries comprised of flight legs that lie within a flight network. Eso (2001) develops an iterative sealed-bid auction for selling excess capacity for an airline company. Vulcano et al. (2002) consider a multi-period version of the Revenue Equivalence Theorem for multi-unit auctions where each individual bidder can be awarded at most one unit. They show that both first and second-price auctions (i.e., the single-unit Vickrey auction) can implement the optimal auction. Pinker et al. (2001) design sequential, multi-unit online Businessto-Consumer auctions to allocate a fixed amount of inventory over a certain number of periods. They consider using bidding information to learn about the valuation of the bidders. However, none of them considers auctions in supply chain settings.

Jin and Wu (2001) show that auctions can serve as a coordination mechanism for supply chains. For supply chain procurement, Beil and Wein (2001) consider a manufacturer who uses a reverse auction to award a contract to a single supplier based on both prices and a set of non-price attributes (e.g., quality, lead time). Chen (2001) studies a firm's optimal procurement strategy for designing a supply chain contract, and an auction mechanism that selects a supplier and determines the procurement quantity and corresponding payment. Most of the studies on multi-unit auctions 
are limited to the case where each bidder can be awarded at most one unit. To the best of our knowledge, the only exception is Gallien and Wein (2000) who design and analyze multi-unit Pay-as-You-Bid procurement auctions in capacity-constrained environments, in which each seller can be awarded multiple units. As the auction mechanism is not truth inducing, they propose a bidding decision support system which provides suppliers competitive information feedback called myopic best response. In our paper, we consider multi-unit auctions in a complex supply chain network. There is no restriction on the quantities a supplier can be awarded. Unlike in classical VCG auctions where only submitted bids (i.e., price schedules) are taken into consideration when making allocation and payment decisions, our VCG auctions incorporate transportation costs into decision making so that the allocation is efficient for the whole supply chain.

\section{Model Introduction}

Consider a single buyer who has requirements, called consumption quantities, for a certain component at a set of geographically diverse locations. As in the standard auction literature, we assume that the buyer has private valuation of the consumption quantities at his demand locations, which form the consumption vector (i.e., demand vector), and he will act strategically to maximize his net utility (i.e., valuation minus acquisition costs). The buyer's private valuation is given by a consumption utility function representing the dollar value of every possible consumption vector to the buyer. This could be, for example, the buyer's expected profit (excluding the acquisition costs which will be determined by the auction) that a consumption vector would bring in. Multiple suppliers, each of whom owns a set of production facilities, are available to satisfy these requirements. Every supplier has a production cost, which can be described as a function of the quantities that she produces at her production locations. Note that the cost function might include a reasonable profit margin for the company. We also assume that each supplier is a rational, self-interested player who is trying to maximize her own profit (i.e. the payments received minus production costs). With general production costs, one can not simply combine the production and transportation costs and treat them as a single cost function. The buyer pays the transportation costs associated with every shipment from a supplier location to a buyer location. So the buyer's acquisition costs include his payments to the suppliers and the transportation costs. We assume that the per-unit transportation cost along each arc of the network is common knowledge to every player. 
We consider three auction mechanisms, $T, S$, and $R$. All three of them require each supplier to submit a bid, a function of the quantity supplied by her, representing her true production costs. Although the three mechanisms have the same payment structure as that of the VCG family, they can lead to very different payments.

Auction $T$ : In this auction, the buyer submits a fixed consumption vector, $\mathbf{q}$, to the auctioneer, who will decide the quantities each supply location will provide to each demand center by minimizing the system production and transportation cost. Under the VCG payment structure, Auction $T$ is truth-inducing and efficient. We investigate how the buyer determines his consumption vector, and show that the consumption vector submitted by the buyer usually does not optimize the total supply chain (i.e., maximize the total net utility of the buyer and the suppliers). Auction $T$ is a direct application of VCG type auction for supply chain procurement. Although truth-inducing and efficient, it may result in unreasonably high payments for the buyer.

Auction $S$ : To demonstrate the benefits of incorporating transportation costs into auctions, we consider Auction $S$ which works the same as Auction $T$ except that the auctioneer decides the production quantities at all supplier locations and the buyer's payments to the suppliers by minimizing the total production cost in the system. Transportation decisions are subsequently made to match the demand and supply at the lowest total transportation cost. It is obvious that Auction $T$ achieves a lower total supply chain cost than Auction $S$. However, the buyer may prefer Auction $S$ under certain circumstances. We found that the typical regular-overtime production cost structure can lead to higher payments in efficient auctions to distort buyer behavior.

Auction $R$ : In this auction, the buyer submits to the auctioneer a utility function, rather than a fixed consumption vector. The auctioneer incorporates this function when deciding allocation and payments to each supplier using the VCG mechanism. The quantities that the buyer will be awarded and his payments are the outcomes of the auction.

Auction $R$ is an enhancement of the standard VCG auctions. It induces truth-telling from the suppliers and guarantees the minimum production and transportation cost for any exchanged quantity vector. Hence, it is efficient. Furthermore, the buyer's payments are always less than or equal to those in Auction $T$ for the same level of consumption. We derive the buyer's optimal strategy assuming the buyer knows the suppliers' production costs (i.e., under complete information) and show that, in general, the buyer will not submit his true consumption utility function. As a result, Auction $R$ typically results in a consumption vector $\mathbf{q}$ that fails to optimize the total supply chain. When the buyer does not have full information about the suppliers' production costs (i.e., under 
partial information), we investigate how the buyer's decision on his bidding function affects his awarded quantities and payments.

Before introducing the detailed auction mechanisms, we first define the notation:

$$
\begin{aligned}
N & =\text { total number of supplier production facilities } \\
K & =\text { number of suppliers } \\
M & =\text { total number of buyer demand centers } \\
N^{k} & =\text { set of production facilities owned by supplier } k \\
k^{n} & =\text { index for the supplier that owns production facility } n \\
q_{m} & =\text { consumption at demand center } m, q_{m}>0 \\
x_{n} & =\text { production quantity at production facility } n \\
y_{n m} & =\text { quantity shipped from production facility } n \text { to demand center } m \\
z_{k m} & =\sum_{n \in N^{k}} y_{n m}, \text { total quantity shipped to demand center } m \text { by supplier } k \\
C_{k}\left(\mathbf{x}_{k}\right) & =\text { production cost function for supplier } k\left(\mathcal{R}^{\left|N^{k}\right|} \rightarrow \mathcal{R}\right) \\
F_{k}\left(\mathbf{x}_{k}\right) & =\text { bidding function from supplier } k\left(\mathcal{R}^{\left|N^{k}\right|} \rightarrow \mathcal{R}\right) \\
\tau_{n m} & =\text { cost for shipping one unit from production facility } n \text { to demand center } m
\end{aligned}
$$

We use boldfaced letters to represent vectors or matrices, whose dimension will be clear from the context. In particular, $\mathbf{x}_{k}=\left(x_{n}: n \in N^{k}\right)$ and $\mathbf{z}_{k}=\left(z_{k m}: 1 \leq m \leq M\right)$. Without loss of generality, $C_{k}(\cdot)$ and $F_{k}(\cdot)$ are assumed to be closed with $C_{k}(\mathbf{0})=F_{k}(\mathbf{0})=0$. All proofs except that of Therem 1 can be found in the online supplement to this paper.

\section{Auction $T$}

In this section, we study Auction $T$ where the buyer submits a fixed consumption vector $\mathbf{q}$ to the auctioneer. Supplier $k$ submits to the auctioneer a bid function $F_{k}\left(\mathbf{x}_{k}\right)$ for supplying $\mathbf{x}_{k}$ units, for

which she incurs a production cost $C_{k}\left(\mathbf{x}_{k}\right), \mathbf{x}_{k} \in \mathcal{R}^{\left|N^{k}\right|}$. The suppliers may or may not see the consumption vector.

As in any auction, the auctioneer will decide the quantities awarded to each of the suppliers $\mathbf{x}_{k}$, the amount transported from each supplier location to each of the buyer locations $y_{n m}$, and the payments made by the buyer to the suppliers. Under Auction $T$, the auctioneer will minimize the 
sum of the accepted bids and the transportation costs, for a given consumption vector $\mathbf{q}$, as

$$
\begin{array}{ll}
\text { Min. } & \sum_{k=1}^{K} F_{k}\left(\mathbf{x}_{k}\right)+\sum_{n=1}^{N} \sum_{m=1}^{M} \tau_{n m} y_{n m} \\
\text { s.t. } & \sum_{n=1}^{N} y_{n m}=q_{m}, \quad m=1, \ldots, M ; \\
& \sum_{m=1}^{M} y_{n m}=x_{n}, \quad n=1, \ldots, N ; \\
& y_{n m} \geq 0, \quad m=1, \ldots, M, \quad n=1, \ldots, N .
\end{array}
$$

Let $\pi(\mathbf{q})$ be the optimal value of the objective function for a given $\mathbf{q}$. Define $\mathcal{Q}=\{\mathbf{q}: \mathbf{q}>\mathbf{0}, \pi(\mathbf{q})<$ $\infty$. We restrict $\mathbf{q} \in \mathcal{Q}$ to ensure sufficient supply capacity. In the case of insufficient capacity, the optimization problem (4.1)-(4.4) may be infeasible or may have an infinite objective function value. Since $F_{k}(\cdot)$ is closed, for any $\mathbf{q} \in \mathcal{Q}$, an optimal solution exists.

Let $\left(\mathbf{x}^{T}, \mathbf{y}^{T}\right)$ be an optimal solution (Note that we use $T$ to indicate that $\left(\mathbf{x}^{T}, \mathbf{y}^{T}\right)$ is a solution of Auction $T)$, and $\pi^{-k}(\mathbf{q})$ be the optimal value of the objective function with the additional constraint $\mathbf{x}_{k}=\mathbf{0}$ (i.e., supplier $k$ does not participate in the auction). The buyer will pay supplier $k$

$$
\psi_{k}^{T}(\mathbf{q})=\pi^{-k}(\mathbf{q})-\pi(\mathbf{q})+F_{k}\left(\mathbf{x}_{k}^{T}\right)
$$

where $\pi^{-k}(\mathbf{q})-\pi(\mathbf{q})$ is the bonus payment made to supplier $k$, representing the value she adds to the system by participating in the auction. The buyer pays supplier $k$ her bid $F_{k}\left(\mathbf{x}_{k}^{T}\right)$, plus her contribution to the system. If the objective function does not include the transportation costs, the payment scheme (4.5) belongs to the VCG mechanism, and all suppliers will bid truthfully irrespective of other players' bids (see Nisan and Ronen (2001), MacKie-Mason and Varian (1994), Vickrey (1961), Clarke (1971) and Groves (1973)). When the transportation costs are included, we show that such auctions belong to the family of Generalized VCG mechanism (defined in Appendix), in which not necessarily all the players receive a VCG type payment (For a formal definition of VCG payments, see Appendix). Groves (1973) shows that truth-inducing is a dominant strategy for every player in a standard VCG mechanism. His argument can be applied to extend the result to the Generailzed VCG family. That is, truth-telling remains a dominant strategy for players receiving VCG payments even if some players in the auction may not receive VCG payments and may not bid truthfully.

Theorem 1 Under the Generalized VCG mechanism, truth-telling is a dominant strategy for players who receive VCG payments. 
The proof can be found in Appendix. In Auction T, the transportation costs in (4.1) can be treated as a bidding function from a dummy bidder (e.g., the buyer) who may or may not receive VCG payments. There are now $K+1$ bidders and the objective is to minimize the total reported costs. Since all bidders except the dummy bidder receive a VCG type payment as in Equation (4.5), by Theorem 1 Auction $T$ induces truth-telling from all suppliers, i.e., $F_{k}\left(\mathbf{x}_{k}\right)=C_{k}\left(\mathbf{x}_{k}\right)$. (Note that this result is not limited to Auction $T$ with transportation costs in the objective function. Other types of costs can similarly be incorporated into allocation and payment decisions in a Generalized VCG auction.) As the unit transportation costs are common knowledge, the buyer will "bid" truthfully. Because we minimize the total transportation and reported production costs in Auction $T$ and the optimal objective value $\pi(\mathbf{q})=\sum_{k=1}^{K} C_{k}\left(\mathbf{x}_{k}^{T}\right)+\sum_{n=1}^{N} \sum_{m=1}^{M} \tau_{n m} y_{n m}^{T}$, the minimum total supply chain cost for meeting the demand $\mathbf{q}$, truth-telling implies that Auction $T$ is efficient.

The total cost incurred by the buyer, $\kappa(\mathbf{q})$, is her total payments to the suppliers plus the transportation costs, i.e.,

$$
\kappa(\mathbf{q})=\sum_{k=1}^{K} \psi_{k}^{T}(\mathbf{q})+\sum_{n=1}^{N} \sum_{m=1}^{M} \tau_{n m} y_{n m}^{T}=\sum_{k=1}^{K} \pi^{-k}(\mathbf{q})-(K-1) \cdot \pi(\mathbf{q}) .
$$

It is interesting to note that, under Auction $T$, the buyer may not choose the $\mathbf{q}$ that optimizes the total supply chain. To see this, we assume that the buyer has complete information (i.e., he can anticipate the bids of the suppliers) and that there exists an increasing and concave Consumption Utility Function, $U(\mathbf{q})$, that specifies the profit (excluding the acquisition costs that will be determined by the auction) the buyer will make by consuming $\mathbf{q}$. The buyer will choose the consumption vector $\mathbf{q}^{B}$ that maximizes his net utility $U(\mathbf{q})-\kappa(\mathbf{q})$. This differs from $\mathbf{q}^{A}$, the consumption vector that maximizes the total net utility of the buyer and the suppliers, $U(\mathbf{q})-\pi(\mathbf{q})$. As $\kappa(\mathbf{q}) \geq \pi(\mathbf{q})$, one might expect $\mathbf{q}^{B} \leq \mathbf{q}^{A}$ (i.e., $q_{m}^{B} \leq q_{m}^{A}, \forall m$ ). This is true when $M=1$, but may not always be true for $M>1$. In fact, even the total quantity of $\mathbf{q}^{B}$ is not always smaller than that of $\mathbf{q}^{A}$. That is, $\sum_{m} q_{m}^{B} \leq \sum_{m} q_{m}^{A}$ does not always hold, as the example in the online supplement to this paper illustrates.

\subsection{Basic properties of Auction $T$}

Here we list several properties of Auction $T$ that will be used repeatedly in the subsequent sections under Assumption 1.

Assumption 1 The production cost function $C_{k}(\cdot)$ and bidding function $F_{k}(\cdot)$ are non-decreasing convex. Furthermore, $C_{k}(\cdot)\left(F_{k}(\cdot)\right)$ is subdifferentiable at points where $C_{k}(\cdot)<\infty\left(F_{k}(\cdot)<\infty\right)$. 
For simplicity, we use $\partial f(\mathbf{x})$ to denote the set of all subgradients of $f(\cdot)$ at $\mathbf{x}$. The convexity of a production cost function implies increasing marginal cost in the quantities produced, which includes the case where the overtime marginal production cost is higher than the marginal cost during regular production time. We will use this assumption for the rest of this paper.

Property $1 \pi(\mathbf{q})$ and $\pi^{-k}(\mathbf{q})$ are increasing convex functions of $\mathbf{q}$.

Property 1 follows directly from the convexity of the production cost functions and linearity of the transportation costs in the convex program (4.1) - (4.4).

Property $\mathbf{2}$ For any optimal solution $\left(\mathbf{x}^{T}, \mathbf{y}^{T}\right)$ for a given $\mathbf{q} \in \mathcal{Q}$, there exist nonnegative Lagrange multipliers, $\mathbf{v} \in \mathcal{R}^{M}$ and $\mathbf{u} \in \mathcal{R}^{N}$ associated with constraints (4.2) and (4.3), such that the following results hold.

1. $\mathbf{u}_{k} \in \partial C_{k}\left(\mathbf{x}_{k}^{T}\right)$ for all $k$.

2. $v_{m}=u_{n}+\tau_{n m}$ if $y_{n m}>0$ and $v_{m} \leq u_{n}+\tau_{n m}$ if $y_{n m}=0$. That is, $v_{m}=\min _{1 \leq n \leq N}\left\{u_{n}+\tau_{n m}\right\}$ for all $m$.

3. $\mathbf{v} \in \partial \pi(\mathbf{q})$ and $\mathbf{v} \in \partial \pi^{-k}\left(\mathbf{q}-\mathbf{z}_{k}^{T}\right)$ where $\mathbf{z}_{k m}^{T}=\sum_{n \in N^{k}} y_{n m}^{T}, 1 \leq m \leq M$.

To understand Property 2, consider the case where $\pi(\cdot), \pi^{-k}(\cdot)$ and $C_{k}(\cdot)$ are differentiable. Then the first two results in Property 2 become

$$
\begin{aligned}
u_{n} & =\frac{\partial C_{k^{n}}\left(\mathbf{x}_{k^{n}}^{T}\right)}{\partial x_{n}}, \\
\frac{\partial C_{k^{n}}\left(\mathbf{x}_{k^{n}}^{T}\right)}{\partial x_{n}}+\tau_{n m} & =\frac{\partial \pi(\mathbf{q})}{\partial q_{m}}, \text { for } y_{n m}^{T}>0 \\
\frac{\partial C_{k^{n}}\left(\mathbf{x}_{k^{n}}^{T}\right)}{\partial x_{n}}+\tau_{n m} & \geq \frac{\partial \pi(\mathbf{q})}{\partial q_{m}}, \text { for } y_{n m}^{T}=0 .
\end{aligned}
$$

That is, suppliers who supply demand center $m$ do so with the same marginal production-plustransportation cost, and suppliers who do not supply demand center $m$ have larger marginal costs at demand location $m$. The third result, $\mathbf{v}=\nabla \pi(\mathbf{q})=\nabla \pi^{-k}\left(\mathbf{q}-\mathbf{z}_{k}^{T}\right)$, implies that the marginal supply chain cost remains the same if we take supplier $k$ and her allocation, $\mathbf{z}_{k}^{T}$, out of the system. These results establish the relationships between the marginal production and transportation costs at any supply location and demand center, and the relationships between the marginal costs at any demand center with and without a supplier (and his bids). 
Since $\mathbf{v}$ may not be unique in general, we define $V(\mathbf{q})$ as the set of all $\mathbf{v}$ that satisfy Property 2. That is, $V(\mathbf{q})=\left\{\mathbf{v}: \exists\right.$ some $\mathbf{u}$ such that $(\mathbf{u}, \mathbf{v})$ satisfies Property 2 for some $\left(\mathbf{x}^{T}, \mathbf{y}^{T}\right)$ given $\mathbf{q} \in \mathcal{Q}\}$. Note that $V(\mathbf{q})$ is nonempty and only depends on $\mathbf{q}$.

Property 3 For any optimal solution $\left(\mathbf{x}^{T}, \mathbf{y}^{T}\right)$ for a given $\mathbf{q} \in \mathcal{Q}$, there exists $\left(\mathbf{x}^{T-k}, \mathbf{y}^{T-k}\right)$ representing an optimal solution without supplier $k$ such that $y_{n m}^{T-k}\left(\mathbf{q}-\mathbf{z}_{k}^{T}\right)=y_{n m}^{T}$ for $1 \leq m \leq M$ and $n \bar{\in} N^{k}$, and $\mathbf{x}_{k^{\prime}}^{T-k}\left(\mathbf{q}-\mathbf{z}_{k}^{T}\right)=\mathbf{x}_{k^{\prime}}^{T}$ for $k^{\prime} \neq k$. That is, if we take supplier $k$ and her awarded quantities $\mathbf{z}_{k}^{T}$ out of the system, then the production and transportation quantities at other suppliers in the auction with fixed demand $\mathbf{q}-\mathbf{z}_{k}^{T}$ remain the same. Furthermore, $\pi^{-k}\left(\mathbf{q}-\mathbf{z}_{k}^{T}\right)$ is the minimum cost for providing $\mathbf{q}-\mathbf{z}_{k}^{T}$ without supplier $k$, and

$$
\pi(\mathbf{q})=\pi^{-k}\left(\mathbf{q}-\mathbf{z}_{k}^{T}\right)+C_{k}\left(\mathbf{x}_{k}^{T}\right)+\sum_{n \in N^{k}} \sum_{m=1}^{M} \tau_{n m} y_{n m}^{T} .
$$

The proof for Property 3 is straightforward and hence, omitted here.

\subsection{The Benefits of Incorporating Transportation Costs into Auctions}

Most literature in multi-unit auctions focuses on the cost of the product, ignoring other costs that will be determined by the outcome of an auction. To examine the benefits of incorporating transportation costs into auctions, we introduce Auction $S$, in which the auctioneer selects production quantities at supplier locations solely based on the suppliers' bids and on demand, and the transportation decisions are made subsequently. For any consumption vector $\mathbf{q}$ the buyer submits, the production quantities at different production facilities are determined by minimizing $\sum_{k=1}^{K} F_{k}\left(\mathbf{x}_{k}\right)$ subject to constraints (4.2)-(4.4). Let $\pi_{S}(\mathbf{q})$ and $\mathrm{x}^{S}$ be the optimal objective value and production vector, and $\pi_{S}^{-k}(\mathbf{q})$ be the counter part of $\pi^{-k}(\mathbf{q})$. The buyer's payment to supplier $k$ in this auction, $\psi_{k}^{S}(\mathbf{q})$, is then given by

$$
\psi_{k}^{S}(\mathbf{q})=\pi_{S}^{-k}(\mathbf{q})-\pi_{S}(\mathbf{q})+F_{k}\left(\mathbf{x}_{k}^{S}\right)
$$

Under this payment structure, the suppliers will still submit their true cost functions and $F_{k}\left(\mathbf{x}_{k}\right)=$ $C_{k}\left(\mathbf{x}_{k}\right)$ for all $k$. The transportation quantities $y_{n m}^{S}$ for all $n$ and $m$ are determined by solving the following optimization problem

$$
\begin{array}{ll}
\text { Min. } & \sum_{n=1}^{N} \sum_{m=1}^{M} \tau_{n m} y_{n m} \\
\text { s.t. } & \sum_{n=1}^{N} y_{n m}=q_{m}, \quad m=1, \ldots, M ;
\end{array}
$$




$$
\begin{aligned}
& \sum_{m=1}^{M} y_{n m}=x_{n}^{S}, \quad n=1, \ldots, N \\
& y_{n m} \geq 0, \quad m=1, \ldots, M, \quad n=1, \ldots, N,
\end{aligned}
$$

and the buyer's total payment, $\kappa_{S}(\mathbf{q})$, will be given by

$$
\kappa_{S}(\mathbf{q})=\sum_{k=1}^{K} \psi_{k}^{S}(\mathbf{q})+\sum_{n=1}^{N} \sum_{m=1}^{M} \tau_{n m} y_{n m}^{S} .
$$

It is obvious that Auction $S$ leads to lower total production costs but higher supply chain costs than Auction T. Our primary interests are the magnitude of the savings by running Auction $T$ and how the buyer's payments differ in two auctions. We show that, under the typical regular-overtime production cost structure, Auction $T$ can lead to payments that are large enough to distort buyer behavior. Intuitively, Auction $T$ is most beneficial when the supplier locations and demand centers are geographically dispersed with distinct production and transportation costs. For example, in a global supply chain suppliers in North America usually have high production costs while suppliers in Asia have lower costs. So in our numerical study, we focus on a supply network where the production and transportation costs are negatively correlated.

Consider a system with three demand centers $(M=3)$ and nine suppliers $(K=9)$. For simplicity, we assume that each demand center has the same fixed demand $q_{m}$. Each supplier owns one production facility $(N=K=9)$ and has a piece-wise linear production cost function. For $n=1, \cdots, 9$,

$$
C_{n}\left(x_{n}\right)= \begin{cases}\alpha_{n} \cdot x_{n}, & \text { if } 0 \leq x_{n} \leq a_{n} \\ \alpha_{n} \cdot a_{n}+\beta_{n} \cdot\left(x_{n}-a_{n}\right), & \text { if } a_{n}<x_{n} \leq b_{n} \\ \infty, & \text { otherwise }\end{cases}
$$

That is, supplier location $n$ has a regular time capacity of $a_{n}$ units with unit production $\operatorname{cost} \alpha_{n}$ and an overtime capacity of $b_{n}-a_{n}$ units with unit production cost $\beta_{n} \geq \alpha_{n}$. We let $a_{n}=40$, total capacity of $b_{n}=60>a_{n}$, unit regular time production cost of $\alpha_{n} \sim N(100,10)$, unit over time production cost of $\beta_{n}=1.5 \alpha_{n}$, unit transportation costs $\tau_{n m} \sim N(50,25)$ where $\left(\alpha_{n}, \tau_{n m}\right)$ are negatively correlated with correlation $\rho \in\{-0.5,-0.25,0\}, m=1, \ldots, M$. For each $(q, \rho)$ pair, we randomly generate a supply network and the costs, and run Auctions $T$ and $S$ to obtain the total production and transportation $\operatorname{cost}\left(\pi_{T}\right.$ and $\left.\pi_{S}\right)$, the total bonus payments $\left(B_{T}\right.$ and $\left.B_{S}\right)$, and the buyer's total payment $\left(\kappa_{T}\right.$ and $\left.\kappa_{S}\right)$. Recall that in Auction $T$, supplier $k$ receives a bonus payment, $\pi^{-k}(\mathbf{q})-\pi(\mathbf{q})$, representing her contribution to the system by participating in the auction. It can 
also be viewed as a profit margin awarded to the supplier above her production costs. The total bonus payments $B_{T}$ is the sum of the bonus payments of all the suppliers in Auction $T$. The total bonus payment in Auction $S, B_{S}$, is calculated in a similar fashion. We repeat these activities 2, 000 times and report the average total supply chain cost $\left(\bar{\pi}_{T}\right.$ and $\left.\bar{\pi}_{S}\right)$, bonus payment $\left(\bar{B}_{T}\right.$ and $\left.\bar{B}_{S}\right)$, and the buyer's total payment $\left(\bar{\kappa}_{T}\right.$ and $\left.\bar{\kappa}_{S}\right)$ in Table 1 . We also included in the table the percentage savings in total supply chain costs $\left(\frac{\bar{\pi}_{S}-\bar{\pi}_{T}}{\bar{\pi}_{T}}\right)$, and buyer's total payments $\left(\frac{\bar{\kappa}_{S}-\bar{\kappa}_{T}}{\bar{\kappa}_{T}}\right)$. As we can see,

\begin{tabular}{|c|c||c|c|c|c|c|c|c|c|}
\hline$q_{m}$ & $\rho$ & $\bar{\pi}_{T}$ & $\bar{\pi}_{S}$ & $\frac{\bar{\pi}_{S}-\bar{\pi}_{T}}{\bar{\pi}_{T}}$ & $\bar{B}_{T}$ & $\bar{B}_{S}$ & $\bar{\kappa}_{T}$ & $\bar{\kappa}_{S}$ & $\frac{\kappa_{S}-\kappa_{T}}{\bar{\kappa}_{T}}$ \\
\hline \hline 50 & -0.50 & 18259.4 & 20422.7 & $11.9 \%$ & 1854.4 & 1203.0 & 20113.7 & 21625.7 & $7.5 \%$ \\
& -0.25 & 17823.8 & 19769.5 & $10.9 \%$ & 2229.0 & 1200.2 & 20052.9 & 20969.7 & $4.6 \%$ \\
& 0.00 & 17398.1 & 19040.4 & $9.4 \%$ & 2553.8 & 1195.7 & 19951.9 & 20236.2 & $1.4 \%$ \\
\hline 75 & -0.50 & 28129.6 & 30061.6 & $6.9 \%$ & 3326.6 & 2284.0 & 31456.2 & 32345.6 & $2.8 \%$ \\
& -0.25 & 27612.7 & 29420.9 & $6.6 \%$ & 4169.0 & 2336.8 & 31781.7 & 31757.7 & $-0.1 \%$ \\
& 0.00 & 27151.8 & 28783.7 & $6.0 \%$ & 4760.4 & 2292.7 & 31912.2 & 31076.4 & $-2.6 \%$ \\
\hline 100 & -0.50 & 38561.6 & 39711.6 & $3.0 \%$ & 5904.2 & 4393.8 & 44465.8 & 44105.5 & $-0.8 \%$ \\
& -0.25 & 38194.9 & 39528.3 & $3.5 \%$ & 7268.1 & 4405.5 & 45463.0 & 43933.7 & $-3.4 \%$ \\
& 0.00 & 37925.7 & 39399.7 & $3.9 \%$ & 8064.5 & 4382.8 & 45990.2 & 43782.5 & $-4.8 \%$ \\
\hline 125 & -0.50 & 49787.6 & 52163.0 & $4.8 \%$ & 15549.2 & 13943.2 & 65336.8 & 66106.1 & $1.2 \%$ \\
& -0.25 & 49625.4 & 51832.1 & $4.5 \%$ & 14608.2 & 13964.4 & 64233.6 & 65796.5 & $2.4 \%$ \\
& 0.00 & 49309.5 & 51400.5 & $4.2 \%$ & 14648.5 & 13947.1 & 63958.1 & 65347.6 & $2.2 \%$ \\
\hline 150 & -0.50 & 62876.1 & 65804.1 & $4.7 \%$ & 23105.7 & 21737.9 & 85981.8 & 87542.0 & $1.8 \%$ \\
& -0.25 & 62456.2 & 65523.7 & $4.9 \%$ & 24355.2 & 21814.0 & 86811.4 & 87337.6 & $0.6 \%$ \\
& 0.00 & 62094.0 & 65238.7 & $5.1 \%$ & 25395.2 & 21768.0 & 87489.2 & 87006.6 & $-0.6 \%$ \\
\hline
\end{tabular}

Table 1: Auction $T$ vs. Auction $S$

Auction $T$ results in savings in total supply chain costs ranging from $3.0 \%$ to $11.9 \%$. As the level of correlation $|\rho|$ increases, the total supply chain costs in both auctions increase monotonically as expected. However, often times the bonus payments in Auction $T$ are much higher than in Auction $S$ so that the buyer actually pays more in Auction $T$. This seems especially true with low correlation between the production and transportation costs. As a result, the bonus portion of the total payments is usually higher in Auction $T$ as shown in Table 2.

Note that a bonus payment to a supplier in a Vickrey type auction measures the supplier's contribution to the system by comparing the total supply chain costs with and without the supplier. The bonus payments can be higher in Auction $T$ than Auction $S$ for the following two reasons.

- While the contribution of a supplier in Auction $S$ is solely based on the production costs, her contribution in Auction $T$ is based on both her production costs and the transportation costs. So a supplier has a higher impact on the total supply chain costs in Auction $T$ than in Auction 
$S$ and hence, receives a higher bonus payment. However, as correlation $|\rho|$ increases, the difference of the total unit production and transportation costs among all suppliers becomes smaller and hence the bonus payments in Auction $T$ decrease. Often times, the difference between the supply chain costs outweighs the difference between the bonus payments, and Auction $T$ leads to both lower total supply chain cost and total buyer's payment.

- Auction $S$ allocates production to the lowest cost suppliers while Auction $T$ allocates production to suppliers with the lowest production and transportation costs. Consequently, more overtime production is likely to be used in determining bonus payments in Auction $T$ and the bonus payments can be significant, especially when the difference between regular time production costs and overtime production costs is big.

The behaviour of the buyer's total payments, which are the sum of the total supply chain cost and the bonus payments, is more complex. When demand is low $\left(q_{m}=50\right.$ in Table 1$)$, there is ample capacity in the system and the difference between the total supply chain costs dominates the difference between the bonus payments. Hence, the buyer pays less in Auction T. As demand increases $\left(q_{m}=75,100\right)$, more overtime capacity is involved in determining the bonus payments in Auction $T$ and the buyer pays more in Auction $T$. As demand continues to increase $\left(q_{m}=125\right)$, a small amount of overtime production is needed to meet the demand in both auctions. The impact of the overtime costs is similar in both auctions and, in general, the buyer pays less in Auction $T$. However, at higher demand level $\left(q_{m}=150\right)$, the transportation costs cause the bonus payments to be much higher in Auction $T$ than Auction $S$, especially when $|\rho|$ is small, and the buyer pays more in Auction T. In most manufacturing systems, per-unit costs are relatively flat when the regular-time capacity of the manufacturing system is not exceeded, while the marginal per-unit production costs grow rapidly when overtime or other measures are required to increase capacity in the short term. As we can see, such cost structure can lead to higher total payments in Auction $T$, making Auction $S$ more preferable to the buyer, although it is less efficient for the overall supply chain. One might think a higher buyer payment is caused by the VCG payment mechanism. However, under mild assumptions, the Revenue Equivalence Theorem indicates that any forward (reverse) auction mechanism that results in an identical allocation will reward the seller (buyer) with the same expected revenue (cost) (Engelbrecht-Wiggans 1988). That is, if there are two other auctions that give the same allocations as in Auction $T$ and $S$ respectively, the difference between the buyer's expected acquisition costs in these two auctions would be the same as that between 


\begin{tabular}{|c|c||c|c|}
\hline$q_{m}$ & $\rho$ & $\bar{B}_{T} / \bar{\kappa}_{T}$ & $\bar{B}_{S} / \bar{\kappa}_{S}$ \\
\hline \hline 50 & -0.50 & $9.22 \%$ & $5.56 \%$ \\
& -0.25 & $11.12 \%$ & $5.72 \%$ \\
& 0.00 & $12.80 \%$ & $5.91 \%$ \\
\hline 75 & -0.50 & $10.58 \%$ & $7.06 \%$ \\
& -0.25 & $13.12 \%$ & $7.36 \%$ \\
& 0.00 & $14.92 \%$ & $7.38 \%$ \\
\hline 100 & -0.50 & $13.28 \%$ & $9.96 \%$ \\
& -0.25 & $15.99 \%$ & $10.03 \%$ \\
& 0.00 & $17.54 \%$ & $10.01 \%$ \\
\hline 125 & -0.50 & $23.80 \%$ & $21.09 \%$ \\
& -0.25 & $22.74 \%$ & $21.22 \%$ \\
& 0.00 & $22.90 \%$ & $21.34 \%$ \\
\hline 150 & -0.50 & $26.87 \%$ & $24.83 \%$ \\
& -0.25 & $28.06 \%$ & $24.98 \%$ \\
& 0.00 & $29.03 \%$ & $25.02 \%$ \\
\hline
\end{tabular}

Table 2: Bonus Portion of the Total Payment in Auction $T$ and Auction $S$

Auction $T$ and $S$. Such a difference is more likely to be determined by the supply network setting and the production cost structure. As a matter of fact, higher buyer payments in Auction $T$ can be avoided when the total regular-time capacity is adequate, or the negative correlation between the production and the transportation costs is high.

Table 2 indicates another interesting point. That is, the bonus portion of the total payments in both auctions is kept in a reasonable range when regular capacity is not very tight or when demand is low. This portion jumps to a much higher level when overtime production is used $\left(q_{m}=125,150\right)$, as the bonus payments increase dramatically because of the big difference between regular-time and overtime production costs. This illustrates the importance of prudent capacity management in markets of manufactured components.

\section{Auction $R$}

As we have shown, the transportation costs can be successfully incorporated into a VCG type auction (Auction $T$ ), which is truth-inducing and efficient for any given q. However, as pointed out by Vickrey (1961), such an auction can be very expensive for the buyer. Consider 10 identical suppliers who meet a total demand of 90 units at a single demand center. Assume that transportation costs are negligible. At each supplier location, $C(x)=F(x)=x$ for $x \leq 9$ and $9+100(x-9)$ for $x>9$. Then it is optimal for all suppliers to produce 9 units at $\$ 1.00$ each and $\pi(90)=\$ 90$. If we remove 
one supplier from the system, the rest of the suppliers will have to produce 9 more units at $\$ 100$ each, which leads to $\pi^{-k}(90)=\$ 981$ for all $k$. The payments from the buyer are then given by $\psi_{k}=\pi^{-k}(90)-\pi(90)+C(9)=\$ 900$ for all 10 suppliers. So the buyer pays a total of $\$ 9,000$ versus the real production cost of $\$ 90$. The reason for such a high payment is that, when capacity is tight and production costs are sharply convex for some suppliers, the total production costs may be significantly different when one of these suppliers is taken out of the system. The bonus payment can be very large, even for something produced at very low costs. In fact, the buyer's net profit, $U(\mathbf{q})-\kappa(\mathbf{q})$, could be negative in some cases in Auctions $T$ and $S$.

Another potential problem with Auction $T$ is that when bidders are asymmetric, i.e., some suppliers have relatively high capacity, removing a supplier with large capacity may result in insufficient capacity and an infinite $\pi^{-k}(\mathbf{q})$ value.

In this section, we propose a new auction mechanism, Auction $R$, that will result in much lower payments for the buyer and yet still induces the suppliers to bid their true costs. In this auction, the buyer submits a function $W(\mathbf{q})$ as a proxy for her true consumption utility, $U(\mathbf{q})$, rather than a fixed consumption vector as in Auction $T . W(\mathbf{q})$ is treated by the auctioneer as if it were the profit (excluding acquisition costs) that the buyer makes by consuming $\mathbf{q}$. We refer to $W(\cdot)$ as the buyer's bidding strategy and make the following assumption about $U(\cdot)$ and $W(\cdot)$.

Assumption $2 U(\mathbf{q})$ and $W(\mathbf{q})$ are increasing concave and closed with $U(\mathbf{0})=0$ and $W(\mathbf{0})=0$. Furthermore, $U(\cdot)(W(\cdot))$ is subdifferentiable at points where $U(\cdot)<\infty(W(\cdot)<\infty)$.

As we will see, by submitting $W(\mathbf{q})$ the buyer is actually reporting a reservation price for each unit he might acquire. It is well-known that one's true reservation price for any unit is the first derivative of her true consumption utility function and is usually NOT identical for all units. Instead, it may well be a decreasing function of the number of units one already has on hand. While in most auctions, the payment to a supplier is determined by bids from the suppliers, in Auction $R$, part or all of the payments will be determined by the buyer's reservation price. This prevents the buyer from making unreasonable payments.

The auctioneer solves the optimization problem $\min \{\pi(\mathbf{q})-W(\mathbf{q})\}$ to determine an optimal consumption vector $\mathbf{q}^{R}$ and associated production quantities $\mathbf{x}^{R}$ and shipments $\mathbf{y}^{R}$. That is, $\mathbf{q}^{R}=$ $\operatorname{argmin}\{\pi(\mathbf{q})-W(\mathbf{q}) \mid \mathbf{q} \geq \mathbf{0}\}$ and $\left(\mathbf{x}^{R}, \mathbf{y}^{R}\right)=\operatorname{argmin}\left\{\sum_{k=1}^{K} F_{k}\left(\mathbf{x}_{k}\right)+\sum_{n=1}^{N} \sum_{m=1}^{M} \tau_{n m} y_{n m} \mid \sum_{m=1}^{M} y_{n m}\right.$ $=x_{n}$ and $\left.\sum_{n=1}^{N} y_{n m}=\mathbf{q}_{m}^{R}, y_{n m} \geq 0\right\}$. If there are multiple minimizers, we assume that the auc- 
tioneer will always choose an optimal consumption vector $\mathbf{q}^{R}$ with the maximum total purchase quantity.

Let $\mathbf{q}^{R^{-k}}$ be the solution to $\min \left\{\pi^{-k}(\mathbf{q})-W(\mathbf{q})\right\}, \Pi(W)=\pi\left(\mathbf{q}^{R}\right)-W\left(\mathbf{q}^{R}\right)$ and $\Pi^{-k}(W)=$ $\pi^{-k}\left(\mathbf{q}^{R^{-k}}\right)-W\left(\mathbf{q}^{R^{-k}}\right)$. Following the VCG payment structure, the payment made to supplier $k$ is

$$
\psi_{k}^{R}(W)=\Pi^{-k}(W)-\Pi(W)+F_{k}\left(\mathbf{x}_{k}^{R}\right)
$$

\subsection{Properties of Auction $R$}

In this section, we show that under (5.8), the suppliers will still bid their costs $C_{k}(\cdot)$, and the buyer pays less for purchasing $\mathbf{q}^{R}$ in Auction $R$ than in Auction $T$. We first introduce the following assumption that guarantees a finite $\mathbf{q}^{R}$ from Auction $R$.

Assumption 3 There exists a finite consumption vector $\mathbf{q}^{\max }$ such that $W(\mathbf{q})=W\left(q_{1}, . ., q_{m}^{\max }, . ., q_{M}\right)$ if $q_{m}>q_{m}^{\max }-1$ for all $\mathbf{q}$, for $W(\cdot)$ satisfying Assumption 2. That is, the buyer associates no utility to any consumption in excess of $q_{m}^{\max }-1$ at demand center $m, 1 \leq m \leq M$.

Under Assumption 3, Auction $R$ belongs to the Generalized VCG family as the buyer can be viewed as a bidder with a bidding function $-W(\mathbf{q})+\sum_{n=1}^{N} \sum_{m=1}^{M} \tau_{n m} y_{n m}$. By Theorem 1, we have the following.

Theorem 2 Under Assumption 3, truth-telling is a dominant strategy for all suppliers in Auction $R$.

That is, $F_{k}\left(\mathbf{x}_{k}\right)=C_{k}\left(\mathbf{x}_{k}\right)$ in Auction $R$. As $\pi\left(\mathbf{q}^{R}\right)=\sum_{k=1}^{K} C_{k}\left(\mathbf{x}_{k}^{R}\right)+\sum_{n=1}^{N} \sum_{m=1}^{M} \tau_{n m} y_{n m}^{R}$ is the minimum total system cost given $\mathbf{q}^{R},\left(\mathbf{x}^{R}, \mathbf{y}^{R}\right)$ minimizes the total supply chain cost for producing and shipping $\mathbf{q}^{R}$.

Interestingly, Auction $R$ can be viewed as a $T$ type auction with fixed consumption vector $\mathbf{q}^{\max }$ and $K+1$ suppliers including the buyer as a dummy supplier who has a production facility with ample capacity co-located with each demand center. These production facilities can only supply their own demand centers and the transportation costs are naturally zero. The buyer is required to bid as a supplier and will bid the following convex function that satisfies Assumption 1,

$$
F_{B}(\mathbf{x})= \begin{cases}0, & \text { if } x_{m}<0 \text { for some } m \\ W\left(\mathbf{q}^{\max }\right)-W\left(\mathbf{q}^{\max }-\mathbf{x}\right), & \text { if } \mathbf{0} \leq \mathbf{x} \leq \mathbf{q}^{\text {max }} \\ +\infty, & \text { otherwise }\end{cases}
$$


where $\mathbf{x}$ represents the vector of the buyer's production quantities. The objective function of this $T$ type auction is $\pi\left(\mathbf{q}^{\max }-\mathbf{x}\right)+F_{B}(\mathbf{x})$, the total reported cost incurred by $K+1$ suppliers for meeting the demand $\mathbf{q}^{\max }$. The auctioneer solves $\min _{\mathbf{0} \leq \mathbf{x} \leq \mathbf{q}_{\max }}\left\{\pi\left(\mathbf{q}^{\max }-\mathbf{x}\right)+W\left(\mathbf{q}^{\max }\right)-W\left(\mathbf{q}^{\max }-\mathbf{x}\right)\right\}$ or $\min _{\mathbf{0} \leq \mathbf{q} \leq \mathbf{q}^{\max }}\left\{\pi(\mathbf{q})+W\left(\mathbf{q}^{\max }\right)-W(\mathbf{q})\right\}$, which is equivalent to $\min _{\mathbf{0} \leq \mathbf{q} \leq \mathbf{q}_{\max }}\{\pi(\mathbf{q})-W(\mathbf{q})\}$, the optimization problem in Auction $R$. Hence the $K$ real suppliers will provide the actual consumption $\mathbf{q}^{R}$ and the dummy supplier, who has ample capacity at each demand center, will supply the remaining $\mathbf{x}_{B}^{R}=\mathbf{q}^{\max }-\mathbf{q}^{R}$.

The auctioneer then solves $\min _{\mathbf{0} \leq \mathbf{x} \leq \mathbf{q}^{\max }}\left\{\pi^{-k}\left(\mathbf{q}^{\max }-\mathbf{x}\right)+W\left(\mathbf{q}^{\max }\right)-W\left(\mathbf{q}^{\max }-\mathbf{x}\right)\right\}$, i.e., $\min _{\mathbf{0} \leq \mathbf{q} \leq \mathbf{q}^{\max }}\left\{\pi^{-k}(\mathbf{q})-W(\mathbf{q})\right\}$ for every $k \in K$, to determine payments to the real suppliers as in a $T$ type auction. However, the dummy supplier does not have a VCG payment. Instead, he will incur the payments to all real suppliers as well as the total transportation cost. As a result, the amounts the real suppliers will produce and ship to the demand locations, the actual payments to the real suppliers, and the total acquisition cost to the buyer in this $T$ auction are exactly the same as those resulting from the original Auction $R$.

If the buyer also submits his true consumption function $U(\mathbf{q})$, Auction $R$ will maximize $U(\mathbf{q})-$ $\pi(\mathbf{q})$ and therefore, optimize the total supply chain. Interestingly, it is usually not optimal for the buyer to do so, as we will show in the next section when we discuss the buyer's optimal bidding strategy. The buyer will usually manipulate $W(\cdot)$ to gain some control over the total acquisition cost

$$
\kappa_{R}(W)=\sum_{k=1}^{K} \psi_{k}^{R}(W)+\sum_{n=1}^{N} \sum_{m=1}^{M} \tau_{n m} \cdot y_{n m}^{R}=\sum_{k=1}^{K} \Pi^{-k}(W)-K \cdot \Pi(W)+\pi\left(\mathbf{q}^{R}\right) .
$$

Since $\mathbf{q}^{R^{-k}}$ minimizes $\left\{\pi^{-k}(\mathbf{q})-W(\mathbf{q})\right\}$,

$$
W\left(\mathbf{q}^{R}\right)-\pi^{-k}\left(\mathbf{q}^{R}\right) \leq W\left(\mathbf{q}^{R^{-k}}\right)-\pi^{-k}\left(\mathbf{q}^{R^{-k}}\right)
$$

for all $k$. Consequently

$$
\kappa_{T}\left(\mathbf{q}^{R}\right)-\kappa_{R}(W)=\sum_{k=1}^{K}\left\{W\left(\mathbf{q}^{R^{-k}}\right)-\pi^{-k}\left(\mathbf{q}^{R^{-k}}\right)-\left[W\left(\mathbf{q}^{R}\right)-\pi^{-k}\left(\mathbf{q}^{R}\right)\right]\right\} \geq 0,
$$

and we have the following theorem.

Theorem 3 Let $\mathbf{q}^{R}$ be a consumption vector that results from $W(\mathbf{q})$ submitted by the buyer in Auction R. For any $W(\mathbf{q})$ that satisfies Assumption 3, The buyer will pay more to purchase the same quantity, $\mathbf{q}^{R}$, in Auction T. That is, $\kappa_{R}(W) \leq \kappa_{T}\left(\mathbf{q}^{R}\right)$. 
From Theorem 3, it appears that the buyer should prefer Auction $R$ to Auction $T$. However as we will see subsequently, Auction $T$ has an important advantage. In Auction $R$, if the buyer does not have complete information about the suppliers' production costs, he cannot predict $\mathbf{q}^{R}$ in advance. In Auction $T$, the quantities acquired by the buyer are known with certainty before the auction begins. Thus, supply uncertainty is an important consideration that favors Auction $T$ over Auction $R$.

\subsection{The Buyer's Optimal Bidding Strategy}

We now explore the optimal $W(\mathbf{q})$ that the buyer would submit with complete information under Auction $R$. We first derive lower bounds for the buyer's total payment $\kappa_{R}(W)$ for a given concave function $W(\mathbf{q})$. We then show that there exists a concave function $W^{*}(\mathbf{q})$ such that $\kappa_{R}\left(W^{*}\right)$ is at the best lower bound and the buyer's profit $U(\mathbf{q})-\kappa_{R}(W)$ is maximized. To establish the lower bounds for the buyer's total payment, we first introduce the following lemma.

Lemma 4 For any optimal solution $\left(\mathbf{x}^{R}, \mathbf{y}^{R}, \mathbf{q}^{R}\right)$ in auction $R$ for a given $W(\cdot)$ satisfying Assumption 3, there exist nonnegative Lagrange multipliers $\mathbf{v} \in \mathcal{R}^{M}, \mathbf{u} \in \mathcal{R}^{N}$ and $\mathbf{u}_{B} \in \mathcal{R}^{M}$ that satisfy the following.

1. $\mathbf{u}_{k} \in \partial C_{k}\left(\mathbf{x}_{k}^{R}\right)$ for all $k$ and $\mathbf{u}_{B} \in \partial F_{B}\left(\mathbf{x}_{B}^{R}\right)$.

2. By Assumption 3, $\mathbf{x}_{B}^{R}>\mathbf{0}$. Since the dummy production facilities only supply their own demand centers, $\mathbf{x}_{B}^{R}=\mathbf{y}_{B}^{R}$ and $\mathbf{v}=\mathbf{u}_{B}$. If we let $r_{m}=\min _{1 \leq n \leq N}\left\{u_{n}+\tau_{n m}\right\}$, then $r_{m}=v_{m}=u_{B m}$ if $q_{m}^{R}>0$ and $r_{m} \geq v_{m}=u_{B m}$ if $q_{m}^{R}=0$.

3. $\mathbf{r} \in \partial W\left(\mathbf{q}^{R}\right), \mathbf{r} \in \partial \pi\left(\mathbf{q}^{R}\right)$, and $\mathbf{r} \in \partial \pi^{-k}\left(\mathbf{q}^{R}-\mathbf{z}_{k}^{R}\right)$.

Lemma 4 indicates that, if $W(\cdot), \pi(\cdot)$, and $\pi^{-k}(\cdot)$ are differentiable, then the marginal utility equals to the marginal supply chain cost with and without supplier $k$ at an optimal solution $\mathbf{q}^{R}$ and $\mathbf{q}^{R}-\mathbf{z}_{k}^{R}$. Since $\mathbf{r}$ may not be unique for any $\mathbf{q}^{R}$, define

$$
\gamma\left(W, \mathbf{q}^{R}\right)=\left\{r: r \text { is defined in Lemma } 4 \text { for some }\left(\mathbf{x}^{R}, \mathbf{y}^{R}, \mathbf{q}^{R}\right)\right\} .
$$

We are now ready to establish the lower bounds of the buyer's total payment for given $W(\cdot)$.

Lemma 5 For any function $W(\cdot)$ satisfying Assumption 3 and the resulting $\mathbf{q}^{R}$ in Auction $R$, $\kappa_{R}(W) \geq \mathbf{r} \cdot \mathbf{q}^{R}$, for any $\mathbf{r} \in \gamma\left(W, \mathbf{q}^{R}\right)$. 
We now show that if $C_{k}(\cdot)$ is strictly increasing for all $k$, there exists $\bar{W}(\mathbf{q})$ such that the buyer's payment $\kappa_{R}(\bar{W})$ is at one of the lower bounds. Bidding at this function, the buyer will pay a uniform price for all the units purchased at the same demand location.

Let $\Gamma(\mathbf{q})=\left\{\mathbf{r}: \mathbf{r} \in \gamma\left(W, \mathbf{q}^{R}\right)\right.$ for some $W(\cdot)$ satisfying Assumption 3 and resulting in $\left.\mathbf{q}^{R}=\mathbf{q}\right\}$. Then for any $\mathbf{r} \in \Gamma(\mathbf{q}), \mathbf{r} \in \partial \pi(\mathbf{q}), \mathbf{r} \in \partial \pi^{-k}\left(\mathbf{q}-\mathbf{z}_{k}^{R}\right)$, and $\mathbf{r} \in \partial W(\mathbf{q})$ for some $W(\cdot)$ by Lemma 4 .

Lemma 6 Assume that $C_{k}(\cdot)$ is strictly increasing for all $k$. Pick any $\overline{\mathbf{q}} \in \mathcal{R}^{M}$, and any $\mathbf{r}$ from $\Gamma(\overline{\mathbf{q}})$. If the buyer submits $\bar{W}(\mathbf{q})=\sum_{m=1}^{M} \min \left\{q_{m}, \bar{q}_{m}\right\} \cdot r_{m}$ in Auction $R$, the resulting consumption vector is $\overline{\mathbf{q}}^{R}=\overline{\mathbf{q}}$ and the buyer's total payment is $\kappa_{R}(\bar{W})=\mathbf{r} \cdot \overline{\mathbf{q}}$. That is, the buyer pays a uniform price $r_{m}$ for all $\bar{q}_{m}$ units shipped to demand center $m$.

We now derive the buyer's strategy $W^{*}(\cdot)$ that maximizes his net utility $U\left(\mathbf{q}^{R}\right)-\kappa_{R}(W)$. As you will see, the structure of $W^{*}(\cdot)$ has the same form as that in Lemma 6 and the buyer needs to find the $(\mathbf{q}, \mathbf{r})$ pair, $\mathbf{r} \in \Gamma(\mathbf{q})$, that maximizes $\{U(\mathbf{q})-\mathbf{r} \cdot \mathbf{q}\}$. For a given $\mathbf{q}$, let $\mathbf{r}^{*}(\mathbf{q}) \in \Gamma(\mathbf{q})$ maximize $\{U(\mathbf{q})-\mathbf{r} \cdot \mathbf{q}\}$. Also, let $\mathbf{q}^{*} \operatorname{maximize}\left\{U(\mathbf{q})-r^{*}(\mathbf{q}) \cdot \mathbf{q}\right\}$ and $\mathbf{r}^{*}=\mathbf{r}^{*}\left(\mathbf{q}^{*}\right)$.

Theorem 7 The optimal function the buyer should submit is $W^{*}(\mathbf{q})=\sum_{m=1}^{M} \min \left\{q_{m}, q_{m}^{*}\right\} \cdot r_{m}^{*}$.

By Theorem 7, the buyer's optimal strategy is determined by two parameters, $\left(\mathbf{r}^{*}, \mathbf{q}^{*}\right)$. To find $\mathbf{r}^{*}$, we need to identify the set $\Gamma(\mathbf{q})$. As you will see in the following lemma, $\Gamma(\mathbf{q})$ is actually equal to the set $V(\mathbf{q})$ defined in Section 4.1 and hence, is nonempty and only dependent on $\mathbf{q}$.

Lemma $8 \Gamma(\mathbf{q})=V(\mathbf{q})$, for any $\mathbf{q} \in \mathcal{Q}$.

If $\pi(\mathbf{q})$ is differentiable for $\mathbf{q} \in \mathcal{Q}$, then $\Gamma(\mathbf{q})=\nabla \pi(\mathbf{q})$ and we can solve for the optimal $\mathbf{q}^{*}$ by maximizing $\{U(\mathbf{q})-\nabla \pi(\mathbf{q}) \cdot \mathbf{q}\}$. Otherwise we can use a differentiable function to approximate $\pi(\mathbf{q})$ and solve for $\mathbf{q}^{*}$ approximately.

With the buyer's optimal strategy specified, we can consider the example in Section 4.2 with 10 identical suppliers under Auction $R$. In that example, the total buyer's payment for providing 90 units under Auction $T$ is $\$ 9,000$ while the total production cost is only $\$ 90$. Recall that there is only one demand center so we can ignore the subscript $m$. To make a meaningful comparison, we assume that $q^{*}=90$. Then the optimal $r^{*}=\$ 1.00$ at $q^{*}=90$, and $W^{*}(q)=\min \left(q, q^{*}\right) r^{*}$. Furthermore, $q^{* R}=q^{*}=90, z_{k}^{* R}=9, q^{* R}-z_{k}^{* R}=81, \pi\left(q^{* R}\right)=\$ 90$, and $\pi^{-k}\left(q^{* R-k}\right)=\$ 81$ for all $k$. So the buyer's total payment is given by

$$
\kappa_{R}\left(W^{*}\right)=\sum_{k=1}^{10}\left[\pi^{-k}\left(q^{* R-k}\right)-W^{*}\left(q^{* R-k}\right)\right]-\sum_{k=1}^{10}\left[\pi\left(q^{* R}\right)-W^{*}\left(q^{* R}\right)\right]+\pi\left(q^{* R}\right)
$$




$$
=\sum_{k=1}^{10}\left[W^{*}(90)-W^{*}(81)\right]+\sum_{k=1}^{10}\left[\pi^{-k}(81)-\pi(90)\right]+\pi(90)=90
$$

which happens to be the minimum supply chain cost.

Interestingly, when the buyer submits his optimal bidding function, $W^{*}(\mathbf{q})$, he actually pays a uniform price $r_{m}^{*}$ for all the units shipped to demand center $m$. This uniform price is his submitted reservation price, as $W^{*}(\mathbf{q})$ is his submitted consumption utility. If $\pi(\cdot)$ is differentiable, $r_{m}^{*}=$

$\frac{\partial \pi\left(\mathbf{q}^{* R}\right)}{\partial q_{m}}$, the marginal supply chain cost at demand center $m$. Under such payment structure, suppliers will try to bid low to get bids accepted, knowing the price is usually greater than their offered bids. Recall that uniform price auctions (e.g., using the first rejected or last accepted bid as the price for each purchased unit) in general do not induce truth-telling from bidders (except for the case where each supplier can be awarded at most one unit) (Ethier et al. 1999). When the buyer follows the optimal strategy, Auction $R$ yields a simple uniform price for each unit shipped to the same demand center and yet, the suppliers still have an incentive to tell the truth.

In summary, Auction $R$ has the following nice properties. (1) It prevents unreasonably high payments in auctions, which may well be a reason for the buyer not to participate. Moreover, it results in lower payments for the buyer than Auction T. (2) The suppliers still have the incentive to submit their true cost functions. (3) When the buyer follows the optimal bidding strategy, his payments have a very simple uniform price structure under full information. (4) For the consumption vector it provides, $\mathbf{q}^{R}$, the total production and transportation cost is minimized and hence, Auction $R$ is efficient. (5) Auction $T$ will work only if any $K-1$ suppliers have sufficient capacity to provide all the demand and $\pi^{-k}(\mathbf{q})$ can be determined. In Auction $R$, the buyer plays a role as a dummy supplier with ample capacity and $\Pi^{-k}(W)$ always exists. (6) The buyer's net profit, $U(\mathbf{q})-\kappa_{R}(W)$, is always nonnegative in Auction $R$, which may not always the case in Auctions $T$ and $S$. That is, the buyer is always better off by participating an $R$ type auction.

\subsection{Auction $R$ under Uncertainty}

So far, we have assumed that the suppliers' production cost functions $C_{k}\left(\mathbf{x}_{k}\right)$ are deterministic and known to the buyer. The buyer's optimal bidding function $W^{*}(\mathbf{q})$ uses this information. However, in reality it is more likely that the buyer has a probabilistic belief about his suppliers' production cost functions, rather than exact knowledge. In addition to facing uncertainty in his payments, he will face uncertainty in his consumption quantities, as we will see from numerical examples later 
on. In this section, we investigate how $W(\cdot)$ affects the mean and variance of the buyer's awarded quantities and his payments.

In general, finding an optimal concave function $W(\cdot)$ under incomplete information is very challenging. Therefore, we will concentrate on functions with the maximum-quantity, reservationprice structure we saw in the previous section. That is, $\bar{W}(\mathbf{q})=\sum_{m=1}^{M} \bar{W}_{m}\left(q_{m}\right)$ where $\bar{W}_{m}\left(q_{m}\right)=$ $\min \left(q_{m}, \bar{q}_{m}\right) \cdot \bar{r}_{m}$ with parameters $\bar{q}_{m}$ and $\bar{r}_{m}$, and we use a computational approach. Utility functions with such structure are easy for practitioners to understand and use. Although the outcome of the auction is uncertain, when the buyer submits $\bar{W}(\cdot)$, he actually sets $\bar{r}_{m}$ as his reservation price for the first $\bar{q}_{m}$ units at demand center $m$ and his reservation price at zero for any unit beyond $\bar{q}_{m}$. So he at least knows that $\overline{\mathbf{q}}$ is an upper bound on the quantities he will be awarded, and that his maximum total payment is bounded from above by $\sum_{m=1}^{M} \bar{q}_{m} \bar{r}_{m}$. For the first $\overline{\mathbf{q}}$ units, sometimes their marginal costs can be too high, even though they are already the lowest from the supply chain, as each supplier is submitting her true cost, so the buyer is actually better off not buying all the $\overline{\mathbf{q}}$ units. If $\bar{r}_{m}$ is set high enough and there is enough capacity to supply $\overline{\mathbf{q}}$, the buyer will purchase exactly $\overline{\mathbf{q}}$ units. In Auction $R, \bar{r}_{m}$, together with bids from suppliers, is sometimes used to determine the payments, so the buyer in general will pay less. If $\bar{r}_{m}$ is sufficiently large, Auction $R$ and a $T$-type auction with fixed demand $\overline{\mathbf{q}}$ will result in the same awards and payments. In fact, Auction $T$ can be viewed as a special case of Auction $R$.

As in Section 4.2, we consider a supply network with nine suppliers and three demand centers. Each supplier owns one production facility and the production cost function $C_{n}(\cdot)$ has a similar structure but with $a_{n} \sim U(30,45), b_{n} \sim a_{n}+U(5,10), \alpha_{n} \sim U(5,25)$ and $\beta_{n} \sim \alpha_{n}+U(5,25)$. As transportation costs are common knowledge, we will create a supply chain network for our numerical study by randomly generating $\tau_{n m} \sim U(2,12)$ once. The data are shown in Table 3 . The data are shown in Table 3 . For simplicity, we assume that the maximum quantity $\bar{q}_{m}$ the buyer wants is the same at each demand center $m$, and we let $\bar{q}=3 \bar{q}_{m}$ denote the sum of the three maximum quantities. Furthermore, we only consider buyer bidding functions with a uniform reserve price $\bar{r}_{m}=\bar{r}$ for all $m$. Thus the only parameters in the buyer's bidding function are $(\bar{q}, \bar{r})$.

For a fixed $(\bar{q}, \bar{r})$ pair, we generate the parameters and run Auction $R$ to obtain a consumption vector and the buyer's total payment. Using this consumption vector as the input, we then run Auction $T$ with the same parameters. We repeat this process 500 times, and calculate the expectation and variance of the purchase quantities and payments under Auctions $R$ and $T$. We repeat the process for various values of $\bar{q}$ and $\bar{r}$. The results are shown in Tables $4-6$. 


\begin{tabular}{|c||ccccccccc|}
\hline \multicolumn{1}{|c||}{} & \multicolumn{8}{|c|}{ Production Facilities $n$} \\
\hline Demand Centers $m$ & 1 & 2 & 3 & 4 & 5 & 6 & 7 & 8 & 9 \\
\hline \hline $\mathbf{1}$ & 11.50 & 4.31 & 8.07 & 6.86 & 10.90 & 9.62 & 6.56 & 2.19 & 10.20 \\
$\mathbf{2}$ & 8.15 & 9.92 & 11.20 & 9.38 & 3.76 & 6.06 & 11.40 & 11.20 & 6.10 \\
$\mathbf{3}$ & 2.58 & 5.53 & 10.10 & 2.10 & 3.39 & 4.03 & 3.99 & 8.04 & 4.72 \\
\hline
\end{tabular}

Table 3: Transportation Cost $\tau_{n m}(\$)$ from Production Facility $n$ to Demand Center $m$

\begin{tabular}{|c||cccccc|}
\hline \multicolumn{1}{|c||}{} & \multicolumn{6}{c|}{ Reserve price $\bar{r}(\$)$} \\
\hline Maximum quantity $\bar{q}$ & 20 & 25 & 30 & 35 & 40 & 45 \\
\hline \hline $\mathbf{7 5}$ & 72.17 & 74.95 & 75 & 75 & 75 & 75 \\
$\mathbf{1 5 0}$ & 130.69 & 148.79 & 150 & 150 & 150 & 150 \\
$\mathbf{2 2 5}$ & 164.50 & 216.88 & 224.90 & 225 & 225 & 225 \\
$\mathbf{3 0 0}$ & 176.82 & 252.83 & 298.04 & 300 & 300 & 300 \\
$\mathbf{3 7 5}$ & 182.49 & 266.44 & 343.97 & 365.64 & 371.87 & 374.18 \\
$\mathbf{4 0 0}$ & 185.28 & 271.06 & 347.80 & 372.21 & 387.54 & 397.90 \\
\hline
\end{tabular}

Table 4: Expected Total Purchase Quantity

In Auction $R$ the buyer's total payments are always lower than those under Auction $T$ for the same purchase quantities, so the entries in Table 6 are always less than or equal to 1 . For any $\bar{q}$, as the reservation price $\bar{r}$ set by the buyer increases, uncertainty in the purchase quantity vanishes, and Auctions $R$ and $T$ become identical. If $\bar{q}$ is large and $\bar{r}$ is small, Auction $R$ buys nearly all units that can be had at the reservation price, and $\bar{q}$ loses its relevance.

For moderate values of $\bar{q}$ and $\bar{r}$, if we carefully decrease $\bar{r}$ and increase $\bar{q}$, the expected purchase quantity can be maintained at a constant level (see Table 4). Costs will go down (see Table 6), but the degree of uncertainty in the total purchase quantity will go up (see Table 5). The buyer will seek an attractive tradeoff between the payments required, and variability in the quantities awarded. To quantify the nature of this tradeoff, we identified a series of pairs $(\bar{q}, \bar{r})$ that result in

\begin{tabular}{|c||cccccc|}
\hline \multicolumn{1}{|c||}{} & \multicolumn{5}{c|}{ Reserve price $\bar{r}(\$)$} \\
\hline Maximum quantity $\bar{q}$ & 20 & 25 & 30 & 35 & 40 & 45 \\
\hline \hline $\mathbf{7 5}$ & 0.117 & 0.015 & 0 & 0 & 0 & 0 \\
$\mathbf{1 5 0}$ & 0.198 & 0.037 & 0 & 0 & 0 & 0 \\
$\mathbf{2 2 5}$ & 0.268 & 0.101 & 0.007 & 0 & 0 & 0 \\
$\mathbf{3 0 0}$ & 0.289 & 0.159 & 0.023 & 0 & 0 & 0 \\
$\mathbf{3 7 5}$ & 0.291 & 0.177 & 0.069 & 0.031 & 0.019 & 0.008 \\
$\mathbf{4 0 0}$ & 0.293 & 0.171 & 0.070 & 0.047 & 0.046 & 0.040 \\
\hline
\end{tabular}

Table 5: Coefficient of Variation of the Total Purchase Quantity 


\begin{tabular}{|c||cccccc|}
\hline \multicolumn{1}{|c||}{} & \multicolumn{6}{c|}{ Reserve price $\bar{r}(\$)$} \\
\hline Maximum quantity $\bar{q}$ & 20 & 25 & 30 & 35 & 40 & 45 \\
\hline \hline $\mathbf{7 5}$ & 0.9759 & 0.9991 & 1 & 1 & 1 & 1 \\
$\mathbf{1 5 0}$ & 0.9544 & 0.9939 & 0.9999 & 1 & 1 & 1 \\
$\mathbf{2 2 5}$ & 0.9441 & 0.9841 & 0.9998 & 1 & 1 & 1 \\
$\mathbf{3 0 0}$ & 0.9411 & 0.9715 & 0.9861 & 0.9991 & 0.9999 & 1 \\
$\mathbf{3 7 5}$ & 0.9413 & 0.9682 & 0.8443 & 0.7284 & 0.7363 & 0.7678 \\
$\mathbf{4 0 0}$ & 0.9437 & 0.9691 & 0.8188 & 0.6653 & 0.6104 & 0.6201 \\
\hline
\end{tabular}

Table 6: The Ratio of the Total Payments between Auction $R$ and Auction $T$

an expected total purchase quantity of 250 units. Figure 1 plots $\bar{q}$, the standard deviation of the total purchase quantity (values on the left axis), and the expectation and standard deviation of the buyer's total payment (values on the right axis). The standard deviation of the total payment is unimodal in our example. It is driven up by the uncertainty in the purchase quantity acquired as $\bar{r}$ decreases, and by the increase in total costs as $\bar{r}$ increases.

The primary tradeoff is between the total payment, and variability in the purchase quantity. The buyer can control the size of his payments via a reservation price, but only by accepting additional risks associated with the amount of material he acquires in the auction, which may cause difficulties in her production planning. In a competitive market, there is not much diffefence between Auctions $T$ and $R$. However, if the market is very asymmetric (i.e, the suppliers have very different cost structures), Auction $R$ may be better. In general, market conditions and buyer preference should be the factors that determine the auction format and reservation prices.

\section{Conclusion}

In this paper, we aim to design multi-unit sealed bid procurement auctions that incorporate both production costs and transportation costs in supply chains involving multiple supplier locations and buyer demand centers. Using insights from classical auction theory, we first construct an optimization-based auction mechanism, Auction $T$, that minimizes the total production and transportation cost for a fixed demand vector. Under VCG payment structure, Auction $T$ induces truth-telling from the suppliers and produces efficient allocation of the buyer's contracts. Furthermore, such a payment based on a supplier's contribution to the system motivates each supplier to improve operational efficiency and lower production costs, increasing her contribution. 


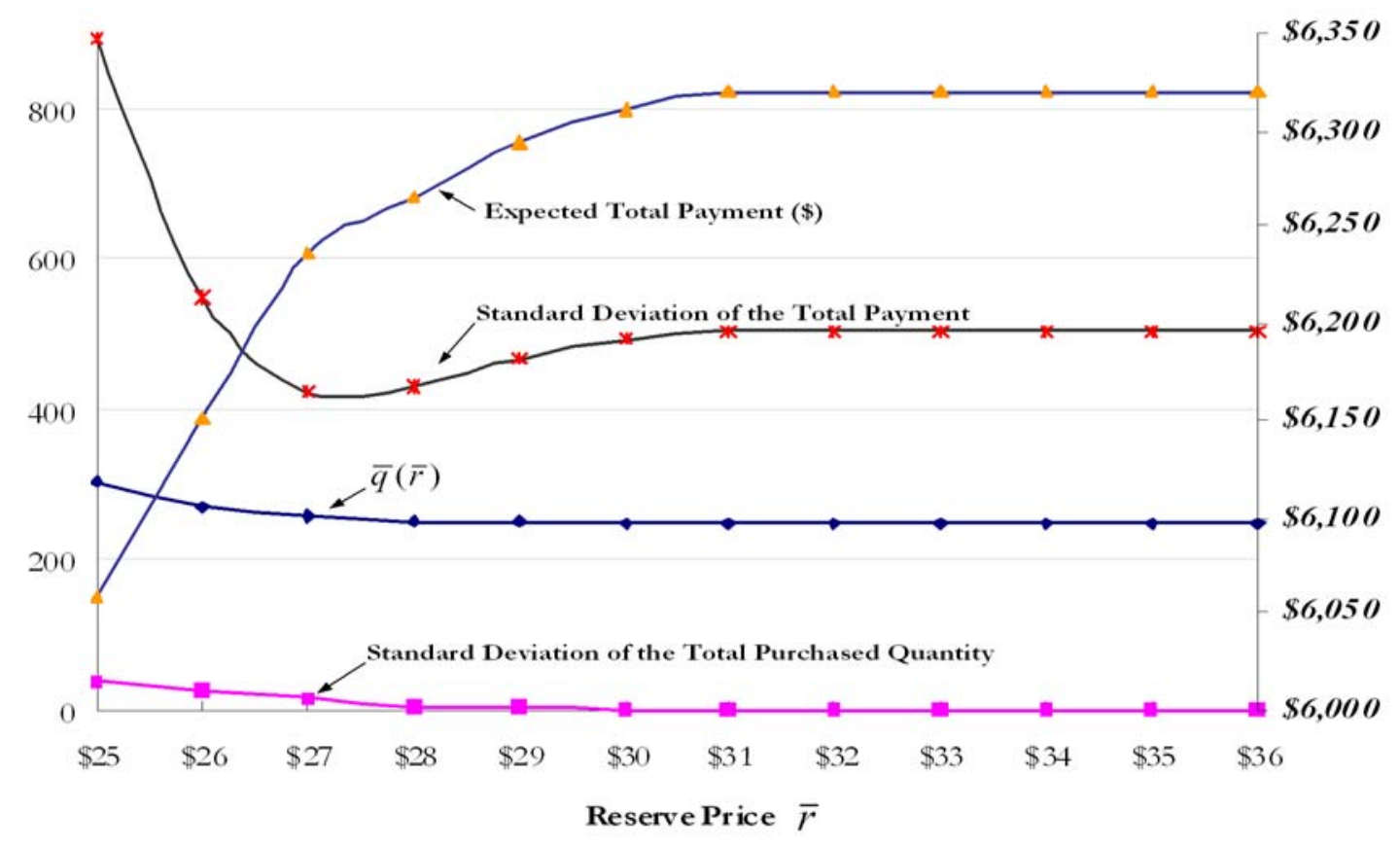

Figure 1: $\bar{q}(\bar{r})$, standard deviation of the awarded quantity, expectation and standard deviation of the buyer's payment

To illustrate the importance of incorporating transportation costs into auctions, we consider Auction $S$, under which the auctioneer awards quantities and makes payment decisions solely based on the suppliers' bids and the demand, and the transportation decisions are made subsequently. Although Auction $S$ induces truth-telling due to its VCG payment structure, numerical examples show that considerable supply chain cost savings can be achieved by running Auction $T$ where both the production and transportation costs are included while making allocation decisions. However, the buyer may favor Auction $S$ due to its lower total payments under certain circumstances. This is because, in Auction $T$, a supplier's contribution is measured not only by her production costs, but also the transportation costs. When the system-wide regular capacity is tight, overtime production and transportation costs together drive the payments up dramatically. The possible higher payments in Auction $T$ can induce the buyer to favor an inefficient auction such as Auction $S$. This illustrates the vital importance of prudent capacity management in markets of manufactured components.

Possible unreasonably high buyer payments in Auction $T$ motivate the development of Auction $R$. In Auction $R$, the buyer also submits a bid, consisting of a dollar-denominated consumption 
utility function that the auctioneer uses when making production and transportation decisions. We show that, under Auction $R$, the buyer will always pay less than he would have paid to obtain the same quantities under Auction T. Consequently, if the buyer can anticipate the bids of the suppliers (complete information), he will always prefer Auction $R$. We derive the buyer's optimal bidding function that maximizes his net utility under complete information. However, if the buyer only has partial information about the suppliers' costs, both the awarded quantities and the buyer's payments are random variables in Auction $R$. This adds a new element of risk that needs to be traded off against the cost savings that the buyer realizes with Auction $R$. In practice a computational approach can be used to make this tradeoff.

This work can be easily extended to VCG auctions with one supplier and multiple buyers. The basic auction, Auction $T$, can also include other types of costs that might arise when parts are purchased in supply chain settings, including fixed production costs, economies of scale in transportation, etc. The basic result that Auctions $T$ and $R$ induce truth-telling and are efficient would easily follow (see the discussion after Equation (4.5)). However, with most of these extensions, our other results may not hold and the required computational effort will increase. Future work also includes auctions in supply chains with multiple suppliers and buyers.

\section{Appendix}

For a general treatment of the Generalized VCG mechanism, we follow the notation of Nisan and Ronen (2001) and explain how it relates to our problem. Suppose that there are $n$ agents. Each agent $i$ has available to it some private information $\theta^{i} \in \Theta^{i}$, termed its type. In a direct revelation mechanism, each agent's strategy is to report its type $t^{i}$, which may or may not be its true type. A mechanism maps the inputs to the auction, a type vector $\mathbf{t}=\left(t^{1}, \ldots, t^{n}\right)$, into an output $o$ belonging to a set of allowed outputs $O$. Each agent $i$ 's preferences are given by a real valued function in terms of some common currency: $v^{i}\left(t^{i}, o\right)$, called its valuation. If the mechanism's output is $o$ and, in addition, the mechanism hands $p^{i}$ units of this currency to agent $i$, then agent $i$ 's utility will be $u^{i}=p^{i}+v^{i}\left(t^{i}, o\right)$. This utility is what the agent aims to optimize. A mechanism $\mathbf{m}=(o, \mathbf{p})$ is composed of two functions of $\mathbf{t}$ : an output function $o()$, and an $n$-tuple of payments $\left(p^{1}(), \ldots, p^{n}()\right)$. We denote $\left(t^{1}, \ldots, t^{i-1}, t^{i+1}, \ldots, t^{n}\right)$ by $\mathbf{t}^{-i}$. That is, $\mathbf{t}=\left(t^{i}, \mathbf{t}^{-i}\right)$.

Definition 1 A direct revelation mechanism $\mathbf{m}=(o(\mathbf{t}), \mathbf{p}(\mathbf{t}))$ belongs to the Generalized $V C G$ family if 
- $o(\mathbf{t}) \in \operatorname{argmax}_{o}\left(\sum_{i=1}^{n} v^{i}\left(t^{i}, o(\mathbf{t})\right)\right)$, and

- $\exists i$ such that

$$
p^{i}(\mathbf{t})=\sum_{j \neq i} v^{j}\left(t^{j}, o(\mathbf{t})\right)+h^{i}\left(\mathbf{t}^{-i}\right)
$$

where $h^{i}()$ is an arbitrary function of $\mathbf{t}^{-i}$. Such agent $i$ is said to receive a VCG payment.

Proof of Theorem 1: Suppose agent $i$ with true type $\theta^{i}$ receives a VCG payment as in (A.1). If truth-telling is not a dominant strategy for the agent, then there exist $t^{i}$ and $\mathbf{t}^{-i}, t^{i} \neq \theta^{i}$ such that,

$$
p^{i}\left(t^{i}, \mathbf{t}^{-i}\right)+v^{i}\left(\theta^{i}, o\left(t^{i}, \mathbf{t}^{-i}\right)\right)>p^{i}\left(\theta^{i}, \mathbf{t}^{-i}\right)+v^{i}\left(\theta^{i}, o\left(\theta^{i}, \mathbf{t}^{-i}\right)\right)
$$

Substituting from (A.1) for $p^{i}\left(t^{i}, \mathbf{t}^{-i}\right)$ and $p^{i}\left(\theta^{i}, \mathbf{t}^{-i}\right)$, this implies that

$$
\sum_{j \neq i} v^{j}\left(t^{j}, o\left(t^{i}, \mathbf{t}^{-i}\right)\right)+v^{i}\left(\theta^{i}, o\left(t^{i}, \mathbf{t}^{-i}\right)\right)>\sum_{j \neq i} v^{j}\left(t^{j}, o\left(\theta^{i}, \mathbf{t}^{-i}\right)\right)+v^{i}\left(\theta^{i}, o\left(\theta^{i}, \mathbf{t}^{-i}\right)\right),
$$

which contradicts $o\left(\theta^{i}, \mathbf{t}^{-i}\right) \in \operatorname{argmax}_{o}\left(\sum_{j \neq i} v^{j}\left(t^{j}, o\left(\theta^{i}, \mathbf{t}^{-i}\right)\right)+v^{i}\left(\theta^{i}, o\left(\theta^{i}, \mathbf{t}^{-i}\right)\right)\right)$, the first condition of the Generalized VCG mechanism. Thus, truth-telling must be a dominant strategy for agent $i$.

Remarks: To relate the notation and the above result to Auction $T$, consider an auction mechanism with $K+1$ players ( $K$ suppliers and one buyer). For the $K$ suppliers, $\theta^{i}=C_{i}\left(\mathbf{x}_{i}\right), t^{i}=F_{i}\left(\mathbf{x}_{i}\right)$, o maps $F_{i}\left(\mathbf{x}_{i}\right)$ into an output of Auction $T, v^{i}(\mathbf{t}, o)=-F_{i}\left(\mathbf{x}_{i}^{T}\right)$, and $h^{i}\left(\mathbf{t}^{-i}\right)=\pi^{-i}(\mathbf{q})$. The buyer can be viewed as a dummy bidder who submits his true transportation costs, which is public information, so that $\theta^{K+1}=t^{K+1}=\left\{\tau_{n m}: n=1, \ldots N, m=1, \ldots, M\right\}$, and $v^{K+1}(\mathbf{t}, o)=-\sum_{n=1}^{N} \sum_{m=1}^{M} \tau_{n m} y_{n m}^{T}$. Then, for any supplier $i$,

$$
p^{i}(\mathbf{t})=-\sum_{\substack{j=1 \\ j \neq i}}^{K} F_{j}\left(\mathbf{x}_{i}^{T}\right)-\sum_{n=1}^{N} \sum_{m=1}^{M} \tau_{n m} y_{n m}^{T}+\pi^{-i}(\mathbf{q})=-\pi(\mathbf{q})+F_{i}\left(\mathbf{x}_{i}^{T}\right)+\pi^{-i}(\mathbf{q})=\psi_{i}^{T}(\mathbf{q})
$$

and truth-telling is still a dominant strategy for all suppliers. This result can be applied to more general settings with other supply chain costs, which can be treated as the value function from the dummy bidder.

Looking at Theorem 1 from another perspective, we can conclude that, if every bidder receives a VCG payment and the outcome $o(\mathbf{t}) \in \operatorname{argmax}_{o}\left(\sum_{i=1}^{n} v^{i}\left(t^{i}, o(\mathbf{t})\right)\right)+w(\mathbf{t})$ where $w()$ is an arbitrary function of $\mathbf{t}$, truth-telling is still a dominant strategy for every bidder. Note that this statement is not as general as Theorem 1, which allows non-VCG payments to a subset of the players. For example, in Auction $R$ the buyer is allowed to bid untruthfully, and truth-telling remains a dominant 
strategy for suppliers (Theorem 2). Theorem 1 extends the classical VCG result to more general settings, in which some but not all of the participants receive VCG payments. As can be seen from the paper, a well designed Generalized VCG auction possesses some nice properties. Although the proof, given here for completeness, is identical to the classical version, we believe this extension opens the door for more variety in mechanism design.

\section{References}

Ausubel, L. and P. Cramton, 1998, "Demand Reduction and Inefficiency in Multi-unit Auctions". Working paper No. 96-07, Department of Economics, University of Maryland.

Beil, D. R. and L. Wein, 2001, "An Inverse-Optimization-Based Auction Mechanism to Support a Multi-Attribute RFQ Process". Management Science, 49(11), 1529-1545.

Chen, F., 2001, "Auctioning Supply Contracts". Working Paper, Graduate School of Business, Columbia University.

Chin, S., "Reverse Auctions Gaining Momentum", Electronics Business Network, March 5, 2003, http://www.ebnews.com.

Chopra, S. and J. Van Mieghem, 2000, "Which E-business is Right for Your Supply Chain?", Supply Chain Management Review, 4(3), 32-40.

Clarke, E. H., 1971, "Multipart Pricing of Public Goods", Public Choice, 11, 17-33.

Cooper, W. L. and R. Menich, 1998, "Airline Ticket Auctions: Revenue Management and the Pivotal Mechanism". Working Paper, Georgia Institute of Technology.

Engelbrecht-Wiggans, R., 1988, "Revenue Equivalence in Multi-object Auctions", Economic Letters, 26, 15-19.

Eso, M., 2001, "An Iterative Online Auction for Airline Seats". Technical report, IBM T. J. Watson Research Center.

Ethier, R., R. Zimmerman, T. Mount, W. Schulze and R. Thomas, 1999, "A Uniform Price Auction with Locational Price Adjustments for Competitive Electricity Markets", Electrical Power and Energy Systems, 21, 103-110.

Gallien, J. and L. Wein, 2001, "A Smart Market for Industrial Procurement with Capacity Constraints", to appear in Management Science.

Groves, T., 1973, "Incentive in Teams", Econometrica, 41, 617-631.

Holmberg, K. and H. Tuy, 1999, "A Production-transportation Problem with Stochastic Demand and Concave Production Costs", Mathematical Programming, 85, 157-179.

Jin, M. and S. D. Wu, 2001, "Supply Chain Coordination in Electronic Markets: Auction and Contracting Mechanisms". Working paper, Department of Industrial and Systems Engineering, Lehigh University. 
Keskinocak, P. and S. Tayur, 2001, "Quantitative Analysis for Internet-Enabled Supply Chains", Interfaces, 31(2), 70-89.

Kisiel, R., "Covisint hit by fall in revenue from auctions", Automotive News Europe, Vol. 7, Issue 15, July 29, 2002.

Klemperer, P., 1999, "Auction Theory: A Guide to the Literature", Journal of Economic Surveys, 13(3), 227-186.

Lucking-Reiley, D., 2000, "Auctions on the Internet: What's Being Auctioned, and How?", Journal of Industrial Economics, 48(3), 227-252.

McAfee, R. P. and J. McMillan, 1987, "Auction and Bidding", Journal of Economic Literature, 25(2), 699-738.

MacKie-Mason, J. K. and H. R. Varian, 1994, "Generalized Vickrey Auctions". Working Paper, Department of Economics, University of Michigan.

Milgrom, P. and R. Weber, 1982, "A Theory of Auctions and Competitive Bidding", Econometrica, 50(5), 1089-1122.

Nisan, N. and A. Ronen, 2001, "Algorithmic Mechanism Design", Games and Economic Behavior, 35(1/2), 166-196.

Pinker, E., A. Seidmann and Y. Vakrat, 2001, "Using Transaction Data for the Design of Sequential, Multi-unit, Online Auctions". Working Paper, W. E. Simon Graduate School of Business Administration, University of Rochester.

Rockafellar, R. T., 1970, Convex Analysis, Princeton University Press, Princeton, New Jersey.

Rothkopf, M. H., 1969, "A Model of Rational Competitive Bidding", Management Science, 15(7), 362-373.

Rothkopf, M. H. and R. M. Harstad, 1994, "Modeling Competitive Bidding: A Critical Essay", Management Science, 40(3), 364-384.

Rothkopf, M. H., T. J. Teisberg, and E. P. Kahn, 1990, "Why are Vickrey Auctions Rare?" Journal of Political Economy, 98(1),94-109.

Segal, I., 2003, "Optimal Pricing Mechanisms with Unknown Demand", The American Economic Review, 93(3),509-529.

Sharp, J. F., J. C. Snyder and J. H. Greene, 1970, "A Decomposition Algorithm for Solving the Multifacility Production-Transportation Problem with Non-linear Production Costs", Econometrica, 38(3), 490-506.

Sullivan, L. and R. Lamb, "Rift Grows Over Pricing in Supply Chain", Electronics Business Network, November 19, 2001, http://www.ebnews.com.

Vickrey, W., 1961, "Counterspeculation, Auctions, and Competitive Sealed Tenders", Journal of Finance, 16, 8-37.

Vulcano, G., G. van Ryzin and C. Maglaras, 2002, "Optimal Dynamic Auctions for Revenue Management". Management Science, 48(11), 1388-1407. 


\section{Online Supplement}

Proof of Property 2: The first two results follow directly from Theorem 28.3 of Rockafellar (1970). We now prove that $\mathbf{v} \in \partial \pi(\mathbf{q})$. The proof for $\mathbf{v} \in \partial \pi^{-k}\left(\mathbf{q}-\mathbf{z}_{k}^{T}\right)$ is similar and is omitted here.

Let $\left(\mathbf{x}^{T}\left(\mathbf{q}^{\prime}\right), \mathbf{y}^{T}\left(\mathbf{q}^{\prime}\right)\right)$ represent an optimal solution for $\mathbf{q}^{\prime} \in \mathcal{Q}$. Then,

$$
\begin{aligned}
\pi\left(\mathbf{q}^{\prime}\right)-\pi(\mathbf{q}) & =\sum_{k=1}^{K}\left[C_{k}\left(\mathbf{x}_{k}^{T}\left(\mathbf{q}^{\prime}\right)\right)-C_{k}\left(\mathbf{x}_{k}^{T}\right)\right]+\sum_{n=1}^{N} \sum_{m=1}^{M} \tau_{n m}\left[y_{n m}^{T}\left(\mathbf{q}^{\prime}\right)-y_{n m}^{T}\right] \\
& \geq \sum_{k=1}^{K} \mathbf{u}_{\mathbf{k}} \cdot\left[\mathbf{x}_{k}^{T}\left(\mathbf{q}^{\prime}\right)-\mathbf{x}_{k}^{T}\right]+\sum_{n=1}^{N} \sum_{m=1}^{M} \tau_{n m}\left[y_{n m}^{T}\left(\mathbf{q}^{\prime}\right)-y_{n m}^{T}\right] \\
& =\sum_{n=1}^{N} u_{n} \sum_{m=1}^{M}\left[y_{n m}^{T}\left(\mathbf{q}^{\prime}\right)-y_{n m}^{T}\right]+\sum_{n=1}^{N} \sum_{m=1}^{M} \tau_{n m}\left[y_{n m}^{T}\left(\mathbf{q}^{\prime}\right)-y_{n m}^{T}\right] \\
& =\sum_{n=1}^{N} \sum_{m=1}^{M}\left(u_{n}+\tau_{n m}\right)\left[y_{n m}^{T}\left(\mathbf{q}^{\prime}\right)-y_{n m}^{T}\right] \\
& =\sum_{n, m: y_{n m}^{T}>0} v_{m}\left[y_{n m}^{T}\left(\mathbf{q}^{\prime}\right)-y_{n m}^{T}\right]+\sum_{n, m: y_{n m}^{T}=0}\left(u_{n}+\tau_{n m}\right) y_{n m}^{T}\left(\mathbf{q}^{\prime}\right) \\
& \sum_{n, m: y_{n m}^{T}>0} v_{m}\left[y_{n m}^{T}\left(\mathbf{q}^{\prime}\right)-y_{n m}^{T}\right]+\sum_{n, m: y_{n m}^{T}=0}^{M} v_{m} y_{n m}^{T}\left(\mathbf{q}^{\prime}\right) \\
& =\sum_{m=1}^{M} v_{m} \sum_{n=1}^{N}\left[y_{n m}^{T}\left(\mathbf{q}^{\prime}\right)-y_{n m}^{T}\right]=\sum_{m=1}^{M} v_{m}\left(q_{m}^{\prime}-q_{m}\right)=\mathbf{v} \cdot\left(\mathbf{q}^{\prime}-\mathbf{q}\right) .
\end{aligned}
$$

The first inequality follows from the first result of this property, and the second inequality from the second result as well as the nonnegativity of $y_{n m}^{T}\left(\mathbf{q}^{\prime}\right)$. The fourth equality holds due to the second result of this property.

Example: Consider a system with 2 suppliers $(K=2)$. Each owns a single production facility $(N=2)$ with production costs $C_{1}(x)=C_{2}(x)=x^{2}$. The buyer has 2 demand centers $(M=2)$ with consumption utility function $U\left(q_{1}, q_{2}\right)=30,000-3\left[100-\left(2 q_{1}+4 q_{2}\right)\right]^{2}$ if $2 q_{1}+4 q_{2} \leq 100$ and $U\left(q_{1}, q_{2}\right)=30,000$ otherwise. That is, there is zero utility associated with each additional unit when $2 q_{1}+4 q_{2}$ is above 100 . Unit transportation costs are $\tau_{11}=\tau_{21}=\tau_{12}=1$, and $\tau_{22}=\infty$. This implies that $\mathbf{y}_{22}^{T}=0$ for all $\mathbf{q}$. Furthermore $q_{2}^{B}=0$ because, if $q_{2}^{B}>0$, then when supplier 1 is removed from the auction, the buyer's transportation cost would be infinite. Thus the buyer would have to make an infinite payment to supplier 1. Consequently, to maximize the buyer's net utility $U\left(q_{1}, q_{2}\right)-\kappa\left(q_{1}, q_{2}\right)$, the buyer will choose $q_{2}^{B}=0$ and $y_{12}^{B}=0$. This leads to

$$
\pi\left(q_{1}, 0\right)=q_{1}^{2} / 2+q_{1}
$$




$$
\pi^{-1}\left(q_{1}, 0\right)=\pi^{-2}\left(q_{1}, 0\right)=q_{1}^{2}+q_{1}
$$

Consequently the buyer's net utility is

$$
U\left(q_{1}^{B}, 0\right)-\kappa\left(q_{1}^{B}, 0\right)=\max \left\{30,000-3\left(100-2 q_{1}\right)^{2}-\left(3 q_{1}^{2} / 2+q_{1}\right)\right\} .
$$

The solution is $\left(q_{1}^{B}, q_{2}^{B}\right)=(44.4,0)$.

By contrast, the supply chain is optimized by solving

$$
\begin{aligned}
U\left(\mathbf{q}^{A}\right)-\pi\left(\mathbf{q}^{A}\right)=\max \quad & 30,000-3\left[100-\left(2 q_{1}+4 q_{2}\right)\right]^{2} \\
& -\left[\left(y_{11}+y_{12}\right)^{2}+y_{21}^{2}+y_{11}+y_{12}+y_{21}\right] \\
\text { s.t. } \quad & y_{11}+y_{21}=q_{1}, \\
& y_{12}=q_{2}, \\
& y_{i j} \geq 0, \quad i=1,2, \quad j=1,2 .
\end{aligned}
$$

The result is $\left(q_{1}^{A}, q_{2}^{A}\right)=(9.63,19.76)$. Note that not only $q_{1}^{B}>q_{1}^{A}$, but also $q_{1}^{B}>q_{1}^{A}+q_{2}^{A}$.

Proof of Lemma 4: Consider the equivalent $T$ auction with fixed demand $\mathbf{q}^{\text {max }}=\mathbf{q}^{R}+\mathbf{x}_{B}^{R}$ resulting from a concave function $W(\cdot)$. Recall that the equivalent $T$ auction has the minimum cost $\Pi(W)=\pi\left(\mathbf{q}^{R}\right)+F_{B}\left(\mathbf{x}_{B}^{R}\right)$. By Property 2, we have the first two results.

To show that $\mathbf{r} \in \partial W\left(\mathbf{q}^{R}\right)$, it suffices to prove that $\mathbf{r} \in \partial F_{B}\left(\mathbf{x}_{B}^{R}\right)$ by the definition of $F_{B}(\cdot)$. Consider any vector $\mathbf{x}_{B}^{\prime} \in \mathcal{R}^{M}$ and, without loss of generality, $\mathbf{0} \leq \mathbf{x}_{B}^{\prime} \leq \mathbf{q}^{\max }$.

$$
F_{B}\left(\mathbf{x}_{B}^{\prime}\right)-F_{B}\left(\mathbf{x}_{B}^{R}\right) \geq \mathbf{u}_{B} \cdot\left(\mathbf{x}_{B}^{\prime}-\mathbf{x}_{B}^{R}\right)=\sum_{m: q_{m}^{R}>0} u_{B m}\left(x_{B m}^{\prime}-x_{B m}^{R}\right)+\sum_{m: q_{m}^{R}=0} u_{B m}\left(x_{B m}^{\prime}-x_{B m}^{R}\right) .
$$

Note that $q_{m}^{R}=0$ implies that $x_{B m}^{R}=q_{m}^{\max }$ and $x_{B m}^{\prime}-x_{B m}^{R} \leq 0$. Since $0 \leq u_{B m} \leq r_{m}$ if $q_{m}^{R}=0$, and $u_{B m}=r_{m}$ if $q_{m}^{R}>0$, we have

$$
F_{B}\left(\mathbf{x}_{B}^{\prime}\right)-F_{B}\left(\mathbf{x}_{B}^{R}\right) \geq \sum_{m: q_{m}^{R}>0} r_{m}\left(x_{B m}^{\prime}-x_{B m}^{R}\right)+\sum_{m: q_{m}^{R}=0} r_{m}\left(x_{B m}^{\prime}-x_{B m}^{R}\right)=\mathbf{r} \cdot\left(\mathbf{x}_{B}^{\prime}-\mathbf{x}_{B}^{R}\right) .
$$

The proof of Property 2 establishes the rest of the lemma.

Proof of Lemma 5: For any $r \in \gamma\left(W, \mathbf{q}^{R}\right)$, let $\left(\mathbf{x}^{R}, \mathbf{y}^{R}\right)$ be an solution associated with it. Rewrite $\kappa_{R}(W)$ as

$$
\kappa_{R}(W)=\sum_{k=1}^{K}\left[\pi^{-k}\left(\mathbf{q}^{R-k}\right)-W\left(\mathbf{q}^{R-k}\right)\right]-K\left[\pi\left(\mathbf{q}^{R}\right)-W\left(\mathbf{q}^{R}\right)\right]+\pi\left(\mathbf{q}^{R}\right) .
$$


Recall that the production and transportation quantities resulting from the $T$ auction for given $\mathbf{q}^{R}$ are the same as those from the $R$ auction with $\mathbf{q}^{R}$ as the output. Also, $z_{k m}^{R}=\sum_{n \in N^{k}} y_{n m}^{R}$, the total quantity shipped to demand center $m$ by supplier $k$. Hence, Property 3 leads to

$$
\begin{aligned}
\pi\left(\mathbf{q}^{R}\right) & =\pi^{-k}\left(\mathbf{q}^{R}-\mathbf{z}_{k}^{R}\right)+F_{k}\left(\mathbf{x}_{k}^{R}\right)+\sum_{n \in N^{k}} \sum_{m=1}^{M} \tau_{n m} y_{n m}^{R}, \\
(1-K) \pi\left(\mathbf{q}^{R}\right) & =\pi\left(\mathbf{q}^{R}\right)-\sum_{k=1}^{K} \pi\left(\mathbf{q}^{R}\right)=-\sum_{k=1}^{K} \pi^{-k}\left(\mathbf{q}^{R}-\mathbf{z}_{k}^{R}\right),
\end{aligned}
$$

and

$$
\kappa_{R}(W)=\sum_{k=1}^{K}\left[\pi^{-k}\left(\mathbf{q}^{R-k}\right)-\pi^{-k}\left(\mathbf{q}^{R}-\mathbf{z}_{k}^{R}\right)+W\left(\mathbf{q}^{R}\right)-W\left(\mathbf{q}^{R-k}\right)\right] .
$$

For any $\mathbf{r} \in \gamma\left(W, \mathbf{q}^{R}\right), \mathbf{r} \in \partial \pi^{-k}\left(\mathbf{q}^{R}-\mathbf{z}_{k}^{R}\right)$ and $\mathbf{r} \in \partial W\left(\mathbf{q}^{R}\right)$ by Lemma 4 . As $\pi^{-k}(\cdot)$ is convex and $W(\cdot)$ is concave,

$$
\begin{aligned}
\pi^{-k}\left(\mathbf{q}^{R-k}\right)-\pi^{-k}\left(\mathbf{q}^{R}-\mathbf{z}_{k}^{R}\right) & \geq \mathbf{r} \cdot\left[\mathbf{q}^{R-k}-\left(\mathbf{q}^{R}-\mathbf{z}_{k}^{R}\right)\right] \\
W\left(\mathbf{q}^{R}\right)-W\left(\mathbf{q}^{R-k}\right) & \geq \mathbf{r} \cdot\left(\mathbf{q}^{R}-\mathbf{q}^{R-k}\right) .
\end{aligned}
$$

Hence,

$$
\kappa_{R}(W) \geq \sum_{k=1}^{K} \mathbf{r} \cdot\left[\mathbf{q}^{R-k}-\left(\mathbf{q}^{R}-\mathbf{z}_{k}^{R}\right)+\left(\mathbf{q}^{R}-\mathbf{q}^{R-k}\right)\right]=\sum_{k=1}^{K} \mathbf{r} \cdot \mathbf{z}_{k}^{R}=\mathbf{r} \cdot \mathbf{q}^{R}
$$

Proof of Lemma 6: If the buyer submits $\bar{W}(\mathbf{q})$, the auctioneer will minimize $\{\pi(\mathbf{q})-\bar{W}(\mathbf{q})\}$ to obtain the consumption vector.

For $\mathbf{r} \in \Gamma(\overline{\mathbf{q}}), \mathbf{r} \in \partial \pi(\overline{\mathbf{q}})$ by the definition of $\Gamma(\cdot)$. By the way $\bar{W}(\cdot)$ is constructed, it is easy to verify that $-\mathbf{r} \in \partial[-\bar{W}(\overline{\mathbf{q}})]$, so that $\mathbf{0}=\mathbf{r}-\mathbf{r} \in \partial \pi(\overline{\mathbf{q}})+[-\partial \bar{W}(\overline{\mathbf{q}})]$. By Theorem 23.8 of Rockafellar (1970), $\partial \pi(\overline{\mathbf{q}})+[-\partial \bar{W}(\overline{\mathbf{q}})] \subset \partial[\pi(\overline{\mathbf{q}})-\bar{W}(\overline{\mathbf{q}})]$. Therefore, $\mathbf{0} \in \partial[\pi(\overline{\mathbf{q}})-\bar{W}(\overline{\mathbf{q}})]$. Since $\pi(\mathbf{q})-\bar{W}(\mathbf{q})$ is a convex program, this indicates that $\overline{\mathbf{q}}$ minimizes $\{\pi(\mathbf{q})-\bar{W}(\mathbf{q})\}$. Let $(\overline{\mathbf{x}}, \overline{\mathbf{y}})$ be a solution associated with $\mathbf{r}$ and $\overline{\mathbf{z}}_{k}=\sum_{n \in N^{k}} \bar{y}_{n m}$. Following the same arguments, $\overline{\mathbf{q}}-\overline{\mathbf{z}}_{k}$ minimizes $\left\{\pi^{-k}(\mathbf{q})-\bar{W}(\mathbf{q})\right\}$ and, for any $\overline{\mathbf{q}}^{R-k}$ that minimizes $\left\{\pi^{-k}(\mathbf{q})-\bar{W}(\mathbf{q})\right\}$,

$$
\pi^{-k}\left(\overline{\mathbf{q}}^{R-k}\right)-\bar{W}\left(\overline{\mathbf{q}}^{R-k}\right)=\pi^{-k}\left(\overline{\mathbf{q}}-\overline{\mathbf{z}}_{k}\right)-\bar{W}\left(\overline{\mathbf{q}}-\overline{\mathbf{z}}_{k}\right) .
$$

Since $C_{k}(\cdot)$ is strictly increasing for all $k, \pi(\mathbf{q})$ is strictly increasing for any $\mathbf{q} \in \mathcal{Q}$. If there exist multiple solutions to $\min \{\pi(\mathbf{q})-\bar{W}(\mathbf{q})\}$, the auctioneer will always choose an optimal consumption 
vector with the largest total purchase quantity as we assumed earlier. We claim that $\overline{\mathbf{q}}^{R}=\overline{\mathbf{q}}$. Suppose there exists an optimal solution with $q_{m}>\bar{q}_{m}$ for some $m$. Then $\bar{W}(\mathbf{q} \wedge \overline{\mathbf{q}})=\bar{W}(\mathbf{q})$, but $\pi(\mathbf{q} \wedge \overline{\mathbf{q}})<\pi(\mathbf{q})$. Thus $\mathbf{q}$ cannot be optimal.

The buyer's total payment is given by

$$
\begin{aligned}
\kappa_{R}(\bar{W}) & =\sum_{k=1}^{K}\left[\pi^{-k}\left(\overline{\mathbf{q}}^{R-k}\right)-\bar{W}\left(\overline{\mathbf{q}}^{R-k}\right)\right]-\sum_{k=1}^{K}[\pi(\overline{\mathbf{q}})-\bar{W}(\overline{\mathbf{q}})]+\pi(\overline{\mathbf{q}}) \\
& =\sum_{k=1}^{K}\left[\bar{W}(\overline{\mathbf{q}})-\bar{W}\left(\overline{\mathbf{q}}^{R-k}\right)\right]+\sum_{k=1}^{K}\left[\pi^{-k}\left(\overline{\mathbf{q}}^{R-k}\right)-\pi(\overline{\mathbf{q}})\right]+\pi(\overline{\mathbf{q}}) \\
& =\sum_{k=1}^{K}\left[\bar{W}(\overline{\mathbf{q}})-\bar{W}\left(\overline{\mathbf{q}}-\overline{\mathbf{z}}_{k}\right)\right]+\sum_{k=1}^{K}\left[\pi^{-k}\left(\overline{\mathbf{q}}-\overline{\mathbf{z}}_{k}\right)-\pi(\overline{\mathbf{q}})\right]+\pi(\overline{\mathbf{q}}) .
\end{aligned}
$$

The last equality follows from Equation (6.9). By Property 3,

$$
\pi(\overline{\mathbf{q}})=\pi^{-k}\left(\overline{\mathbf{q}}-\overline{\mathbf{z}}_{k}\right)+C_{k}\left(\overline{\mathbf{x}}_{k}\right)+\sum_{n \in N^{k}} \sum_{m=1}^{M} \tau_{n m} \bar{y}_{n m} .
$$

Summing over $k$, we have

$$
\pi(\overline{\mathbf{q}})=-\sum_{k=1}^{K}\left[\pi^{-k}\left(\overline{\mathbf{q}}-\overline{\mathbf{z}}_{k}\right)-\pi(\overline{\mathbf{q}})\right]
$$

and

$$
\kappa_{R}(\bar{W})=\sum_{k=1}^{K}\left[\bar{W}(\overline{\mathbf{q}})-\bar{W}\left(\overline{\mathbf{q}}-\overline{\mathbf{z}}_{k}\right)\right]=\sum_{m=1}^{M}\left(\sum_{n \in N^{k}} r_{m} \cdot \bar{y}_{n m}\right)=\mathbf{r} \cdot \overline{\mathbf{q}} .
$$

That is, the buyer pays a uniform price $r_{m}$, for all the units shipped to demand center $m$.

Proof of Theorem 7: If the buyer submits $W^{*}(\mathbf{q})$, by Lemma 6, the resulting consumption vector is $\mathbf{q}^{* R}=\mathbf{q}^{*}$, the buyer's total payment is $\kappa_{R}\left(W^{*}\right)=\mathbf{r}^{*} \cdot \mathbf{q}^{*}$, and the buyer pays a uniform price $r_{m}^{*}$ for all units shipped to demand center $m$. For any $W(\mathbf{q})$ resulting in $\mathbf{q}^{R}$ as the output of Auction $R$, by Lemma 5 ,

$$
U\left(\mathbf{q}^{R}\right)-\kappa_{R}(W) \leq U\left(\mathbf{q}^{R}\right)-\mathbf{r} \cdot \mathbf{q}^{R}
$$

for all $\mathbf{r} \in \gamma\left(W, \mathbf{q}^{R}\right) \subseteq \Gamma\left(\mathbf{q}^{R}\right)$. For any $\mathbf{r} \in \Gamma\left(\mathbf{q}^{R}\right)$,

$$
U\left(\mathbf{q}^{R}\right)-\mathbf{r} \cdot \mathbf{q}^{R} \leq U\left(\mathbf{q}^{R}\right)-r^{*}\left(\mathbf{q}^{R}\right) \cdot \mathbf{q}^{R} \leq U\left(\mathbf{q}^{*}\right)-\mathbf{r}^{*} \cdot \mathbf{q}^{*}
$$

So

$$
U\left(\mathbf{q}^{R}\right)-\kappa_{R}(W) \leq U\left(\mathbf{q}^{*}\right)-\mathbf{r}^{*} \cdot \mathbf{q}^{*}
$$

That is, in Auction $R$, the buyer's utility is maximized by submitting $W^{*}(\mathbf{q})$. 
Proof of Lemma 8: For any given $\mathbf{r} \in \Gamma(\mathbf{q}), \mathbf{r} \in \gamma\left(W, \mathbf{q}^{R}\right)$ for some $W$ satisfying Assumption 3 with $\mathbf{q}^{R}=\mathbf{q}$. The associated $\left(\mathbf{x}^{R}, \mathbf{y}^{R}, \mathbf{u}, \mathbf{u}_{B}\right)$ satisfies Lemma 4 and hence, $\left(\mathbf{x}^{R}, \mathbf{y}^{R}, \mathbf{u}, \mathbf{r}\right)$ satisfies Property 2. Therefore, $\mathbf{r} \in V(\mathbf{q})$.

For any given $\mathbf{v} \in V(\mathbf{q})$, there exists $\left(\mathbf{x}^{T}, \mathbf{y}^{T}, \mathbf{u}\right)$ satisfying Property 2 . Hence, $\mathbf{v} \in \partial \pi(\mathbf{q})$ and $\mathbf{v} \in \partial \pi^{-k}\left(\mathbf{q}-\mathbf{z}_{k}^{T}\right)$. We can construct $\bar{W}(\mathbf{q})$ as defined in Lemma 6 with parameter $\mathbf{v}$ instead of a $\mathbf{r}$ from set $\Gamma(\mathbf{q})$. By similar arguments in the proof of Lemma $6, \bar{W}(\cdot)$ results in $\overline{\mathbf{q}}^{R}=\mathbf{q}$ and hence, $\mathbf{v} \in \gamma(\bar{W}, \mathbf{q})$. Therefore, $\mathbf{v} \in \Gamma(\mathbf{q})$ and $\Gamma(\mathbf{q})=V(\mathbf{q})$ for any $\mathbf{q} \in \mathcal{Q}$. 